Nasalisation in the production of Iraqi Arabic pharyngeals

Ghada Khattab, Jalal Al-Tamimi, Wasan Alsiraih

Newcastle University

Corresponding author: Ghada Khattab (ghada.khattab@newcastle.ac.uk)

Postal address: Speech and Language Sciences, King George VI Building, Queen Victoria Road, Newcastle University, Newcastle upon Tyne, NE1 7RU, UK. Tel: +44 191 2086583

Email addresses: ghada.khattab@newcastle.ac.uk; jalal.al-tamimi@newcastle.ac.uk; wasansalamsiraih@yahoo.com 


\title{
Nasalisation in the production of Iraqi Arabic pharyngeal
}

\begin{abstract}
Aim: This paper presents an auditory and acoustic investigation of pharyngeal consonants in Iraqi Arabic (IA). While the contested place and manner of articulation of these sounds have been the subject of investigation in many studies, the focus here is novel: we set out to investigate the extent to which pharyngeals in IA are accompanied by auditory nasalisation and if so, how widespread the effect is across oral and nasal contexts.

Methods: Auditory and acoustic properties of nasalisation were investigated in target words with oral, nasal, and pharyngeal environments as produced by nine male speakers of IA.

Results: When combined with oral consonants, pharyngeals exhibit little or no nasalisation; however, when pharyngeals are combined with nasals, they exhibit various degrees of nasalisation, sometimes beyond what is found for a nasal environment alone. This is especially so for voiced pharyngeals, which display more nasalisation than their voiceless counterparts. A Principle Component Analysis combining all the acoustic correlates examined demonstrates a definite contribution of pharyngeals to the presence of nasalisation. Conclusion: The epilaryngeal constriction and variability in the articulation of pharyngeals are thought to be responsible for the nasalisation effect and may act as potential drivers for sound change in IA pharyngeals.
\end{abstract}

\section{Pharyngeal consonants: a diverse category of sounds}

Interest in the pharyngeal consonants $/ \mathrm{h} /$ and $/ \mathrm{S} /$ has been prominent due to their varying realisations across dialects, especially in the case of the voiced target (Ghazeli, 1977; Laradi, 1983; Butcher and Ahmad, 1987; Hassan, Esling, Moisik and Crevier-Buchman, 2011; Heselwood, 2007; Heselwood and Al-Tamimi, F., 2011; Esling, 1999, 2005). While these sounds are officially classified as pharyngeal fricatives on the IPA chart, their reported manner of articulation varies from open approximation to full closure, and their reported 
place of articulation varies from pharyngeal to laryngeal or even epiglottal. Some researchers maintain that the voiceless and voiced counterparts share the same manner of articulation, which ranges from approximant in some studies (e.g. Catford, 1977; Shahin, 2002; Heselwood, 2007; Yeou and Maeda, 2011) to aryepiglottic trilling in others (e.g. Hassan et al, 2011). Other researchers maintain that $/ \hbar /$ is indeed a voiceless fricative but differ in their description of /S/, which has been labelled as: a voiced approximant (Obrecht, 1968; Ingham, 1982; Esling, 1999; Esling, 2005); a tight approximant (Heselwood, 2007); a glottal stop (AlAni, 1970; MacCurtain, 1981); or an epiglottal stop [?] (Esling, 1999; Esling, 2005; Edmondson, Esling, Harris and Huang, 2005; Edmondson, Padayodi, Hassan and Esling 2007).

Apart from dialectal variation, one potential reason for the variation in the description of the voiced pharyngeal is in the separate actions of the epiglottis and the tongue root. In a fiberscopic and acoustic analysis of Hebrew pharyngeals (Laufer and Condax, 1979; 1981), the epiglottis was found to be moving backwards separately from the root of the tongue during the articulation of both pharyngeals. The epiglottis either formed a narrow constriction with the pharyngeal wall or a complete closure with it. This led Laufer and Condax (1979) to conclude that the epiglottis is "an active and independent articulator" in producing pharyngeal consonants. In their study of Moroccan Arabic pharyngeals, Zeroual, Esling and Crevier-Buchman (2008) further identify two separate actions in the epiglottis: one between the tip of the epiglottis and the posterior pharyngeal wall (epiglotto-pharyngeal articulation), and the other between the base of the epiglottis, the aryepiglottic folds, and the tip of the arytenoids (aryepiglottic sphincter or epilaryngeal articulation). This last action led Esling (2005) to suggest that the aryepiglottic folds are the active articulators in the production of Arabic pharyngeals, while the epiglottis is the passive one. He therefore advocated for pharyngeal consonants to be labelled epiglottal rather than pharyngeal, in order to better 
reflect their place of articulation, with retraction of the tongue and raising of the larynx for efficient laryngeal sphinctering (Esling, 2005).

\section{Pharyngeal consonants in Iraqi Arabic}

Early investigations of the pharyngeals In Iraqi Arabic were mostly based on impressionistic accounts and noted a difference in place and manner between $/ \hbar /$ and $/ \mathrm{\varsigma} /$. $/ \hbar /$ was described as a voiceless fricative with a pharyngeal constriction, which became voiced in intervocalic position (Al-Ani, 1970; McCarthy and Raffouli, 1964). The most common allophone for /9/ was reported to be a glottal stop, especially in initial and final position, while it was realised as a glide inter-vocalically (Al-Ani, 1970). As articulatory investigations have become more widespread, the role of the epiglottis and the larynx in the production of pharyngeals has come to light, but disagreement still exists regarding the exact mechanism(s) involved in the production of this complex set of sounds. Using X-ray images, MacCurtain (1981) observed the tip of the epiglottis blocking the pharyngeal passage and having no direct contact with the tongue root, providing evidence that the epiglottis is used as an articulator in producing the /9/ in IA. MacCurtain (1981) also reported shortening of the vestibular folds and raising of the larynx. Butcher and Ahmad's (1987) acoustic and aerodynamic study further confirmed the role of the epiglottis and laryngeal tension. The authors noted that the pharyngeal area is not suitable for producing a fricative, suggesting that $/ \mathrm{h} /$ is either an approximant with a general constriction of the pharynx, a raised larynx and a lowered velum (an issue we will go back to below) or a stop with a constriction formed at the epiglottis or the glottis. As for $/ \mathrm{S} /$, any additional constriction of the pharynx was thought to be accompanied by one at the larynx, leading the air to be completely interrupted at one or both positions (Butcher and Ahmad, 1987). In a more recent study, Edmondson et al (2007) found Iraqi $/ \mathrm{h} /$ to exhibit extreme laryngeal constriction of the aryepiglottic folds at the top of the epilaryngeal tube. Pharyngeal / $/$ /, on the other hand, was mostly realised as a voiced approximant, with some 
instances of slight trilling. Aryepiglottic trilling is thought to be present in all voiced pharyngeals (Hassan et al, 2011), with geminates displaying the highest degree of aryepiglottic fold movement given the repeated trilling cycles.

The finding of velum lowering in pharyngeals by Butcher and Ahmad's study above is intriguing, and one that typically receives less attention in the study of this category of sounds. In the section below, we examine potential articulatory mechanisms which might lead to nasal coupling as an indirect consequence of pharyngeal articulation.

\section{Nasalisation in Pharyngeal consonants}

A less frequently examined aspect of pharyngeal production is accompanying nasalisation. In fact, although nine of the 13 chapters from an edited volume on "Instrumental Studies in Arabic Phonetics" (Hassan and Heselwood, 2011) deal with guttural and emphatic (pharyngealised) consonants, none investigates nasalisation or refers to it as a feature of pharyngeal production. The link between nasal and pharyngeal consonants has been sporadically referred to since early studies on Ancient West Arabian by Rabin (1951) and South-Ethiopic languages by Hetzron (1969), though few have tried to investigate this experimentally (e.g. Butcher and Ahmad, 1987; Ghazeli, 1977; Laradi, 1983).

One of the first studies associating pharyngeal consonants with nasalisation is by Hetzron (1969), who investigated South-Ethiopic languages and found non-etymological $n$ 's in words where the consonantal root used to contain a pharyngeal (e.g. Amharic [and] 'one', originating from /ahad/, and Western Gurage [anqyä] 'after' originating from $/ h^{\mathrm{h}} \mathrm{q}^{(\mathrm{w})} /$, Hetzron (1969: 71)). Hetzron noted that these $n$ 's were homorganic with the following consonant and believed that their appearance was connected with the nasalisation of the original pharyngeals. His observation was confirmed following personal correspondence with Delattre, who had carried out an exploratory x-ray study of pharyngeals in Iraqi Arabic (later published in 1971) and found that vowels following these sounds were nasalised. Delattre 
attributed the nasalisation to the extreme lowering of the uvula towards the root of the tongue in the production of the pharyngeals $/ \mathrm{S} /$ and $/ \hbar /$, forcing a separation of the velum and rhinopharyngeal wall.

Another interesting connection between pharyngeals and nasals was made in Mahdi's (1985) descriptive study of the spoken Arabic of Basra. Mahdi referred to the occurrence of

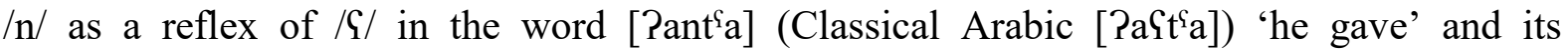
derivations in Basri Arabic; he gave it the Arabic term al-'istinta:' [PalPistint'a?] (Mahdi, 1985: 74) and assumed that it happened due nasalisation spread from / $/$ / being blocked by the following stop, surfacing as a nasal consonant (see alternative account by Rabin, 1951, who suggests 'anța [Pant ${ }^{\complement} a$ ] as the older word, but who still agrees that the [Pant $\left.{ }^{\complement} a\right]-\left[\right.$ PaAt $^{\complement} a$ ] alternation is due to the pharyngeal having a nasal element in its articulation).

In small-scale experimental investigations around the same time, Arabic pharyngeals were indeed found to be produced with accompanying velum lowering and/or nasal airflow, but this did not always lead to audible nasalisation and there was notable across-speaker variation. For instance, in his study of back consonants in various Arabic dialects, Ghazeli (1977) looked at the articulatory characteristics (using cinefluorographic film) and the oralnasal airflow (using an Electro Aerometer) of pharyngeal articulations in his own production of pharyngeal consonants in Tunisian Arabic. He noted lowering of the velum, but did not find any nasal airflow accompanying it; further investigation revealed that while the lower tip of the velum moves down the pharyngeal wall, the upper posterior tip remains in contact with it, even if over a small area.

In a physiological and articulatory study of pharyngealisation in various Arabic dialects (using video-endoscopic and video-fluorographic recordings, spectrographic analysis, palatographic and airflow measurements), Laradi (1983) looked closely at $/ \mathrm{h} /$ and $/ \mathrm{S} /$ in Libyan Arabic; she found that the tongue was slightly lowered and the root of the tongue 
retracted towards the back of the pharynx, causing a narrowing at the oropharyngeal cavity and creating a constriction between the epiglottis and the pharyngeal wall. She also noted larynx raising and jaw and velum lowering. However, like Ghazeli, Laradi (1983) noted that a small part of the velum was still partially in contact with the nasopharynx, even if the rest of it seemed to be away from it. The contact was more pronounced for $/ \mathrm{S} /$ than $/ \hbar /$, and indeed /h/ showed variable nasal airflow. Nasal airflow also tended to vary across the speakers and dialects investigated (she also looked at Yemeni and Iraqi) but no firm conclusions could be drawn due to the small number of speakers and no audible nasalisation was heard regardless of whether or not velum lowering was noted.

Finally, in a study of the acoustic and aerodynamic characteristics of pharyngeal consonants in Iraqi Arabic, Butcher and Ahmad (1987) note inconsistency across speakers, with only one participant showing nasal airflow in connection to $/ \mathrm{h} /$ and mainly in initial position, suggesting context- and speaker-specific patterns.

So why should pharyngeal consonants be accompanied by nasalisation? Laver (1980) observes that pharyngeal constriction is attained by two lingual settings: pharyngealised voice, which covers constrictions of the middle of the pharynx, versus laryngopharyngealised voice, which covers constrictions of the lower pharynx and upper larynx. Both or either constriction may be accompanied by a backward and slightly downward movement of the main body of the tongue, leading the velum to be pulled downwards due to pressure from the anterior faucal pillar and palatoglossus muscles (Dixit, Bell-Berti and Harris, 1987). This action, however, is not automatic and in fact even in nasal production not all speakers activate the palatoglossus to cause velum lowering (Bell-Berti, 1993; Sampson, 1999). Laver further notes that it is difficult to auditorily distinguish between the two pharyngeal constrictions because other physiological and acoustical events tend to accompany any constriction in the pharynx; these include larynx lowering, which leads to a 
breathy-like phonatory setting, unless the muscles around the larynx are braced (Laver, 1980). The latter must be the case for Arabic pharyngeals, since the experimental studies on Arabic pharyngeals reviewed above found the larynx to rise rather than lower in the production of these consonants. Similar observations have been made since by Heselwood and Al-Tamimi, F. (2011) using nasoendoscopy and videofluoroscopy, who show larynx raising in the production of Jordanian pharyngeals, alongside involvement of the epiglottis and pharyngeal contraction.

Another potential reason for nasalisation is the laryngeal constriction which accompanies pharyngealisation. In laryngeal constrictions, a high laryngeal airflow caused by the open glottis leads to an upward shifts in all formants of neighbouring vowels while lowering their amplitude and increasing their bandwidth, especially for $F 1$ (Ohala and Ohala, 1993; Ohala, 1975); this, together with the presence of pole-zeros in the higher frequencies of spectrum due to the greater glottal opening, yields a perceptual effect of nasality which can further be adopted by speakers who might deliberately adopt a physiologically nasal articulation in the production of these neighbouring vowels (Matisoff, 1975; Ohala, 1975; Sampson, 1999). In fact, laryngeals have often been reported as being the target of nasalisation processes (Botma, 2004; Cohn, 1993; Ohala and Ohala, 1993). These processes involve a combination of a lowered velum and a constriction behind the uvula, leading them to be labelled as 'nasalised laryngeals' (Botma 2004: 279). Botma notes that audible nasalisation is more prominent in glottal fricatives and continuants, while complete glottal stops may mask nasalisation or delay it until the release. Another potential source of nasal perception in pharyngeals is the action of the aryepiglottic folds, which can move up and forward to meet the epiglottis and produce an incomplete constriction, creating laryngeal friction (Esling, 1999, Heselwood; 2007).

\section{Aim of the current study}


The various mechanisms taking place in the articulation of Arabic pharyngeal consonants lead to potential velum lowering and/or auditory nasalisation; the latter seems to stem from constrictions that do not just involve the root of the tongue and the upper pharyngeal area, but also epilaryngeal constrictions in lower pharyngeal area involving the aryepiglottic folds and the tip of the arytenoids (Butcher and Ahmad, 1987; Edmondson et al, 2007; Esling, 2005; Heselwood and Al-Tamimi, F., 2011; MacCurtain, 1981). The number and the degree of these constrictions may either lead to the creation of sub-cavities between the constrictions or to the generation of high laryngeal airflow, both of which can produce acoustic consequences which yield an auditory impression of nasalisation. Since any or all of the articulatory events listed above can (co-)occur in pharyngeal articulation, the combined effect of all these constrictions is still poorly understood. The articulatory complexity, combined with the limitation and difficulty in obtaining articulatory data, motivated an acoustic study as a starting point.

In this study we look at the acoustic effects of pharyngeals on surrounding vowels, especially with regards to the frequencies and amplitudes of the harmonics around $F 1$, where the effect of a laryngeal airflow and a pharyngeal constriction seem to be compatible. The focus of the study is not on the presence or absence of nasal coupling but rather on the auditory and acoustic manifestations of pharyngeal articulations in Iraqi pharyngeals. The aim is to investigate the degree to which auditory nasalisation accompanies the production of pharyngeals in IA and its potential spread to non-pharyngeals environments. There are two general motivations for this study. First, given that IA pharyngeal consonants have been consistently found to have lower pharyngeal and laryngeal constriction (Section 2), and given the likely acoustic effects of these constrictions, we hypothesised that they will be prone to nasalisation. Second, we are aware of anecdotal references to IA as having a nasalised voice quality, which further motivates and investigation of whether nasalisation has indeed spread 
beyond pharyngeal environments. This is the first quantitative examination of nasalisation both as a potential feature of pharyngeals in IA and as a voice quality setting colouring the speech of a speech community. Given that breathy phonation is sometimes masked as nasalisation (Chen, 2000), we first examined nasalisation and breathiness ratings in our auditory analysis and then we included acoustic measures of breathiness alongside nasalisation in an attempt to disentangle the two, especially in the production of pharyngeals.

Before moving on to the methodology, we briefly review acoustic studies of nasalisation in order to inform our acoustic analysis.

\section{The acoustic investigation of nasalisation}

A number of studies have looked at the acoustic manifestations of nasal and nasalised sounds, which are mainly due to the effect of oral-nasal coupling (e.g. Amino and Osanai, 2012 on Japanese; Beddor, 1983; 2007 on various languages; Chen, 1995 and Chen, Slifka and Stevens, 2007 on American English; Chen, 1997 on American English and French; Chen, 2000 on Chinese; Glass and Zue, 1985 on American English; Hawkins and Stevens, 1985 on American English; Ohala, 1975 on various languages; Trittin and de Santos y Lléo, 1995 on Spanish). The effect of the nasal cavity leads to the presence of extra poles in the spectrum in addition to the oral formants (as well as to zeros due to cancelling out of certain resonances) (e.g. Beddor, 1983; Chen et al., 2007; Ohala, 1975). A host of acoustic measurements comparing vowels in oral and nasal environments have been investigated for capturing the effect of nasalisation, e.g. decrease in vowel intensity, upwards/downwards shifts in $F 1$ and F2 frequencies (depending on original vowel quality but also depending on which study is consulted), increase in $F 1$ bandwidth $(B 1)$, decrease in $F 1$ amplitude (A1), and presence of extra poles $(P 0$ and $P 1)$ in the spectrum and the resulting differences in amplitude between these peaks and those of $F 1$. What makes these measures difficult to adopt as reliable measures of nasalisation is that a) none of them is present at all times, b) extra poles (and 
zeros) are present in oral vowels too (e.g. due to passive velar lowering, especially in low vowels, or speaker style/physiology, see Berger, 2007 and Klatt and Klatt, 1990), and c) some measures have yielded conflicting results partly due to vowel-, language- and speakerspecific differences (Sampson, 1999). Here we focus on a handful of indices that were relatively effective in measuring nasalisation in this study and that enabled the comparison of nasal, oral and pharyngeal environments.

Nasalised vowels exhibit two types of spectral peaks in addition to vowel formants: a spectral peak $(P 1)$ normally develops around $1 \mathrm{kHz}$ (with a range between 790 and $1100 \mathrm{~Hz}$ and an average of 950Hz) (e.g. Chen 1995; Chen et al., 2007; Fry, 1979; Hawkins and Stevens, 1985; House, 1957; House and Stevens, 1956); another nasal peak (P0) can also be found between 250 and $450 \mathrm{~Hz}$ (e.g. Chen, 1997; Chen et al., 2000; Fujimura and Lindqvist, 1971; Maeda, 1982) (Figure 1). The location of the poles depends on vowel quality, and there are conflicting results regarding whether they occur below or above $F 1$ frequency in various vowels contexts (e.g. Fujimura and Lindqvist, 1971 vs Maeda, 1982). Poles and zeros also occur in non-nasalised (oral) vowels, but the poles in nasalised vowels are more noticeable because the increase in amplitude of $P 1$ compared to $A 1$ is especially prominent and/or there is a deepened valley between the poles and $F 1$ (Glass and Zue, 1985: 1571).

The addition of poles at certain frequencies, coupled with the absorption of energy at others, results in a widened $F 1$ bandwidth $(B 1)$ and a decrease in $F 1$ amplitude $(A 1)$ due to the larger area of damping created by the nasal cavity (Figure 2). The effect sometimes exhibits decreases in the overall vowel intensity and an increased bandwidth (e.g. Beddor, 1983; 2007; Chen, 1995; Fujimura and Lindqvist, 1971; Hawkins and Stevens, 1985). Using the formula $A 1-P 1$ (where $A 1$ is the amplitude of $F 1$ and $P 1$ the amplitude of the extra peak), Chen's study of nasalisation in American English vowels suggests that a small difference (less than $10 \mathrm{~dB}$ ) is considered as indicative of the vowel being nasal. In a later study, Chen 
(1997) notes the role of the two extra poles $(P 0$ and $P 1)$ in signalling nasalisation across languages (Figure 1). A comparison between American English and French showed that in both languages the difference in amplitude between $A 1$ and the extra peaks ( $A 1-P 1$ or $A 1-P 0)$ distinguished between oral and nasalised vowels, with the ranges being language-specific (the mean of $A 1-P 1$ ranged from 10 to $15 \mathrm{~dB}$ for English and from 9 to $12 \mathrm{~dB}$ for French; and the mean of $A 1-P 0$ ranged from 6 to $8 \mathrm{~dB}$ for English and from 3 to $9 \mathrm{~dB}$ for French). These differences were considered to be due to the wider velopharyngeal opening in French compared with English, potentially due to the contrastive role of nasalisation in the French and the contextual role in English.
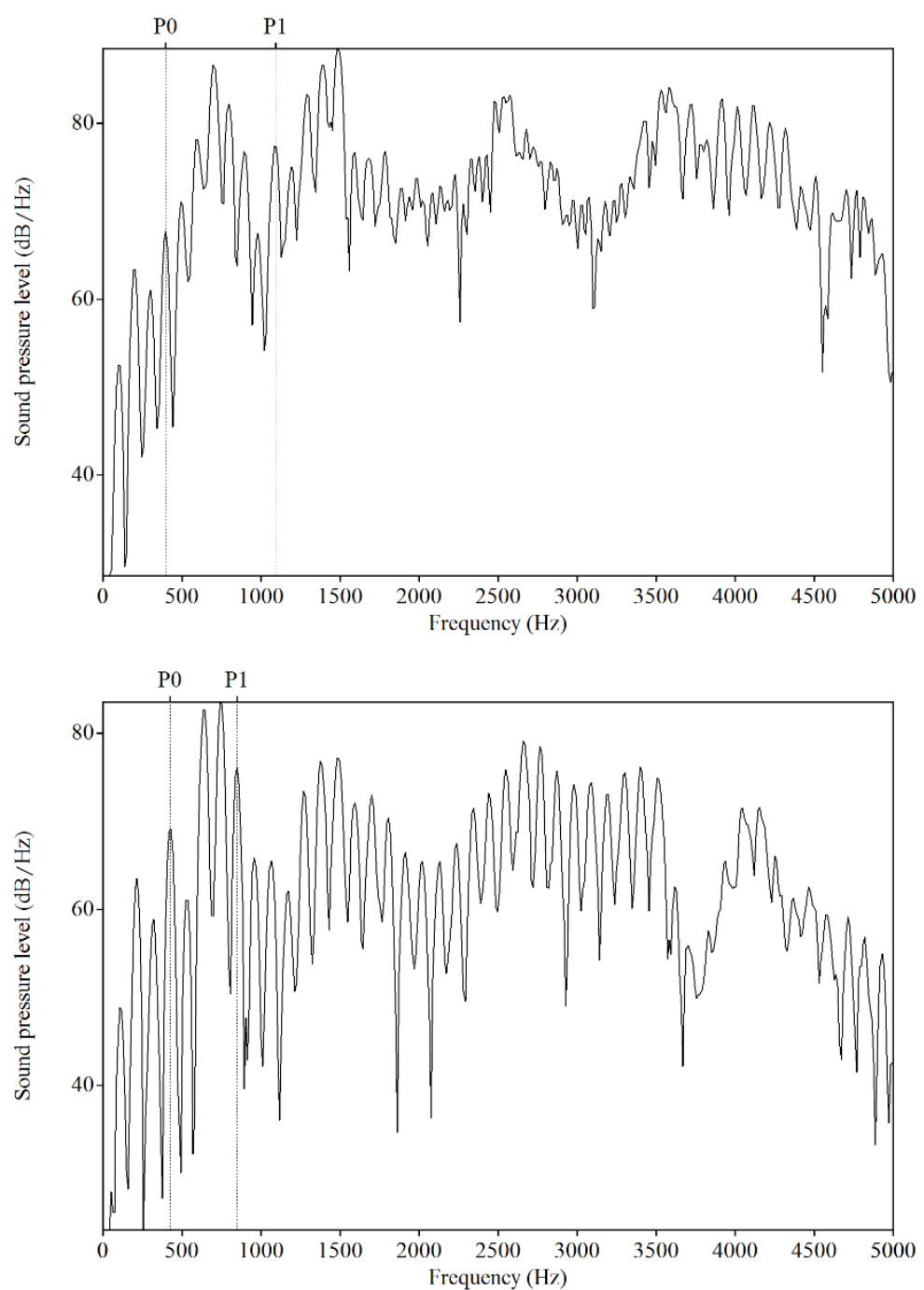

Figure 1: (a) DFT spectrum of an oral vowel in the word '[da:s]' he stepped compared with (b) DFT spectrum of a nasalised vowel in the word '[na:m]' he slept as produced by a male Iraqi speaker from the current study. The position of $\mathrm{PO}$ and P1 in both is indicated. The amplitudes of A1-P1 and A1-P0 are 
greater for the oral vowel than the nasal vowel (though only A1-P0 was used in the analyses for this particular vowel).
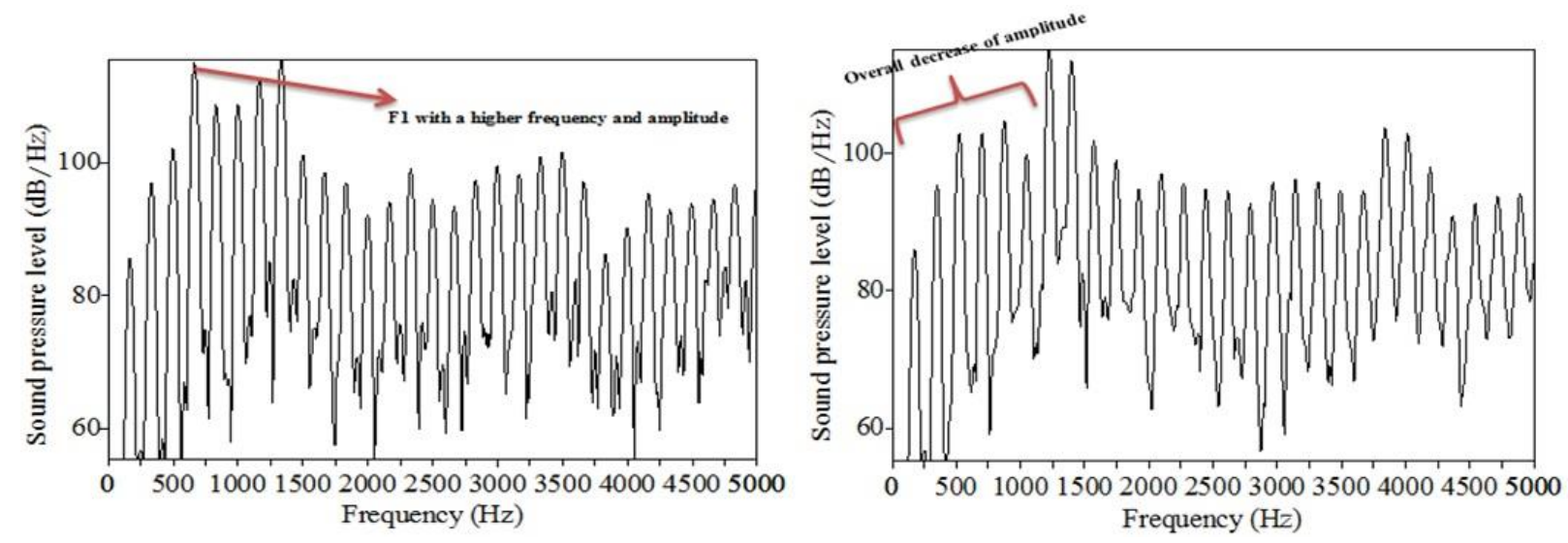

Figure 2: Spectra taken from the midpoint of the vowel [a:] as produced by a male Iraqi speaker, first in isolation (on the left) and then in the word maat [ma:t] 'he died' (on the right), showing a decrease in amplitude and frequency of $A 1$ (on the $3^{\text {rd }}$ harmonic) relative to the same vowel in isolation. The peak on the $5^{\text {th }}$ harmonic was considered as $P 1$.

The A1-P1 measure is normally more suited for high vowels given the distance between $F 1$ and $F 2$ frequencies, and $A 1-P 0$ for low vowels given the distance below $F 1$ (e.g. Chen, 1995, 1997, 2000; Chen et al., 2000; Chen et al., 2007). However, vowel breathiness can also affect the values of both $A 1-P 0$ and $B 1$ due to an increase in the amplitude of the first harmonic (H1) of the fundamental frequency (e.g. Fry, 1979; Keating and Esposito, 2007; Laver, 1980; Klatt and Klatt, 1990). It is therefore important to look at parameters of breathiness in order to disentangle these from nasalisation. The difference between amplitude of the first $(H 1)$ and second $(H 2)$ harmonic $(H 1-H 2)$ has been found useful for characterising differences in phonation types or glottal constrictions (Figure 3), with a higher $H 1$ than $H 2$ typically signalling breathiness, while a lower or equal one can be indicative of creak (e.g. Chen, 2007; Keating and Esposito, 2007; Kuang and Keating, 2012; Ladefoged, 2003). Similarly, a comparison of the amplitude of the first harmonic $(H 1)$ with that of the first formant $(A 1)$ has proven to be a successful measure of phonation types in many languages (e.g. Fry, 1979; Laver, 1980, 1994; Klatt and Klatt, 1990; Trittin and de Santos y Lléo, 1995). This measure is directly related to the first formant bandwidth $(B 1)$, which in turn is related to 
the posterior glottal opening, i.e. if a speaker has a posterior glottal opening, then first formant bandwidth will be increased. Glottal opening causes modifications to the spectrum such as the increase of $B 1$ which is caused by added energy loss at the glottis (Hanson and Chuang, 1999: 1064). Speakers with high $H 1-A 1$ values, showing weaker $F 1$ peaks and more aspiration noise, are therefore assumed to have relatively large glottal openings extending beyond the vocal processes, suggesting breathiness (Hanson, 1996; Hanson and Chuang, 1999).

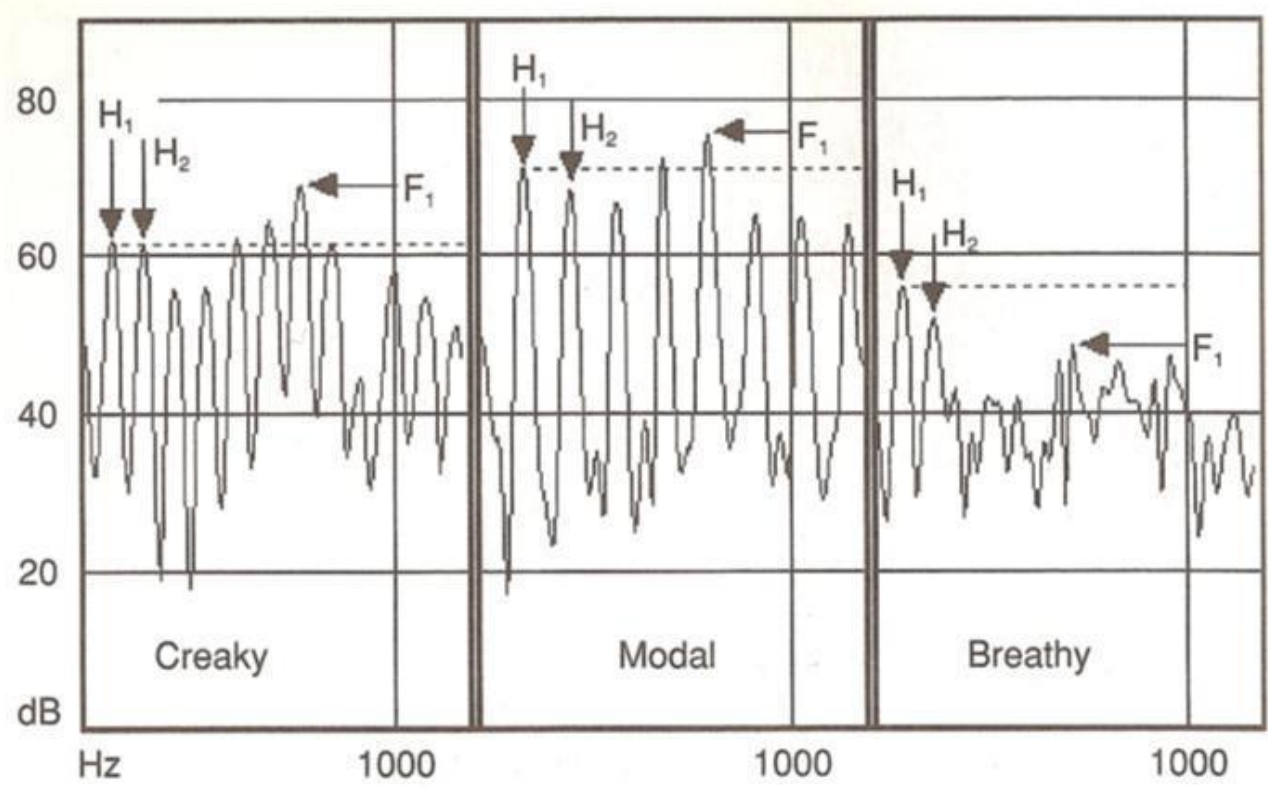

Figure 3: Spectra of creaky, modal and breathy [a] vowel in San Lucas Quiavini Zapotec, a language spoken in Mexico. The dashed line marks the intensity of $\mathrm{H1}$ in each spectrum. The relation between $\mathrm{H} 1$ and $H 2$ was found to correlate with the type of phonation: creaky when $H 1$ is almost equal to $H 2$, modal when $H 1$ is about $2 \mathrm{~dB}$ higher than $H 2$ and breathy when $H 1$ is about $4 \mathrm{~dB}$ higher than $H 2$ (Figure from Ladefoged, 2003: 180).

\section{Method}

\subsection{Speakers}

Nine Iraqi male participants were recruited from a population of postgraduate students who had been living in the UK for no more than two years in order to minimise the influence of English on their L1 speech patterns. All participants spoke Iraqi Arabic (IA) as their native language and had been raised in one of three main dialect areas: the Northern (Upper Iraq) 
qeltu dialect of Mosul, the Central gelet dialect of Baghdad, and the Southern gelet dialect of Basra (see Blanc, 1964 for dialect groups in Iraq). For the purpose of this study, the dialectal origin of the speakers is included for information, but dialect is not included as a variable (though see Alsiraih, 2013 for more detailed results on dialectal differences within IA). The speakers were aged between 30 and 50 years and had been living in their home-towns all their lives except for the time they studied in the UK. The speakers had no known voice disorders or vocal-nasal apparatus problems.

\subsection{Stimuli and procedure}

The participants read a randomised list of 122 monosyllabic real words with a CVC structure embedded in the carrier sentence 'quulu ... sit marrat' (Say .... six times); the vowel in each case was one of the five long vowels /i:, e:, a:, u:, s:/ or one of the four short vowels /I, e, $\Lambda$, $\mho /$, and the surrounding consonants were chosen from one of three consonant types: a nasal stop $/ \mathrm{m}, \mathrm{n} /$, a pharyngeal consonant $/ \mathrm{f}, \mathrm{h} /$, or any other consonant (Table 1 ). The utterances with the long vowels were repeated, with the second production consisting of the isolated long vowels embedded in the carrier sentence (in order to facilitate their production).

The vowels were examined in five main environments: First, words with a nasal context were needed to investigate the effect of true/target nasals on surrounding vowels. These were considered as control environments. Initial and final nasal consonants were included in order to measure whether nasalisation effect was progressive or regressive, leading to a nasal-nasal, nasal-oral or oral-nasal context. Second, words with a pharyngeal consonant in either position were included in order to investigate whether these consonants in IA have accompanying nasalisation as is suggested in the literature, and if so, whether the nasalisation effect is similar to that of nasal contexts both in terms of degree and direction of influence. These included pharyngeal-oral and oral-pharyngeal contexts only (there are hardly any CVC words with both initial and final pharyngeal consonants). Third, words with 
both a nasal and a pharyngeal consonant (i.e. nasal-pharyngeal and pharyngeal-nasal) were included in order to investigate whether nasalisation increases in such an environment compared with a nasal-nasal, a nasal-oral or oral-nasal environment. The fourth context consisted of two oral consonants (oral-oral) in order to investigate whether nasalisation and/or other voice quality features (e.g. breathy phonation) are still present in such cases where no nasal consonant is present. For similar reasons, the last context included long vowels in isolation, though still produced within the carrier sentence frame. These last two environments, oral-oral and isolation, were considered as control environments, just like the nasal ones.

When taking into consideration each of the pharyngeal and each of the word positions, the five main environments encompass the 12 individual contexts detailed in Table 1 . The original aim was to elicit a target word for each of the consonant and vowel contexts listed above ( 9 vowels in 12 consonant environments each), but the criterion to use real words made it hard to either find words for all these contexts or to keep them after piloting, as some were rejected by participants. For this reason and in order to keep the focus on the consonantal environments, we grouped the vowel contexts into two main groups, high (including highmid) versus low (including low-mid), as these proved useful for the acoustic measures detailed below. The data were recorded in mono, 16-bit, $44.1 \mathrm{kHz}$ using an Edirol R09 solidstate recorder with a Sony Electret Condenser microphone placed in front of the seated speakers at a 45 degree angle. A total of 1854 tokens were auditorily and acoustically analysed.

Table 1: CVC contexts, their abreviations and examples.

\begin{tabular}{|c|c|c|c|}
\hline Context & Abbreviation & Example1 & Example2 \\
\hline nasal-vowel-nasal & $\mathrm{n}-\mathrm{n}$ & $/$ naam/ 'he slept' & $\begin{array}{c}/ \mathrm{mi}: \mathrm{m} / \text { 'the letter } \mathrm{m} \text { in } \\
\text { Arabic' }\end{array}$ \\
\hline nasal-vowel-oral & $\mathrm{n}-\mathrm{o}$ & $/ \mathrm{ma}: \mathrm{s} /$ 'diamond' & $/ \mathrm{mall} /$ 'he got bored' \\
\hline oral-vowel-nasal & $\mathrm{o}-\mathrm{n}$ & $/ \mathrm{xa}: \mathrm{m} /$ 'raw material' & $/ \mathrm{di}: \mathrm{n} /$ 'religion' \\
\hline
\end{tabular}




\begin{tabular}{|c|c|c|c|}
\hline $\begin{array}{c}\text { nasal-vowel- } \\
\text { pharyngeal }\end{array}$ & $n-\hbar$ & /nu:ћ/ 'Noah' & /na:h/ 'he wept' \\
\hline $\begin{array}{c}\text { nasal-vowel- } \\
\text { pharyngeal }\end{array}$ & $n-€$ & /no:S/ 'type' & /maS/ 'with' \\
\hline $\begin{array}{l}\text { pharyngeal-vowel- } \\
\text { nasal }\end{array}$ & $\hbar-n$ & /hakk/ 'he scratched' & $\begin{array}{c}\text { /ho:m/ 'a group of prey } \\
\text { birds' }\end{array}$ \\
\hline $\begin{array}{l}\text { pharyngeal-vowel- } \\
\text { nasal }\end{array}$ & S-n & /ৎっ:n/ 'a helpful hand' & /Samm/'uncle' \\
\hline $\begin{array}{l}\text { pharyngeal-vowel- } \\
\text { oral }\end{array}$ & $\hbar-\mathrm{O}$ & /ha:1/ 'situation' & /har/ 'hot' \\
\hline $\begin{array}{l}\text { pharyngeal-vowel- } \\
\text { oral }\end{array}$ & S-O & / Sadd/ 'he coutned' & /Gi:d/ 'bring back' \\
\hline $\begin{array}{l}\text { oral-vowel- } \\
\text { pharyngeal }\end{array}$ & $0-\hbar$ & $\begin{array}{c}\text { /la:h/ 'was able to } \\
\text { reach' }\end{array}$ & /zi:ћ/ 'line’ \\
\hline $\begin{array}{l}\text { oral-vowel- } \\
\text { pharyngeal }\end{array}$ & $0-\uparrow$ & /dzu:S/ 'hunger' & /ba:S/ 'he sold' \\
\hline oral-vowel-oral & O-O & /ba:b/ 'door' & /dub/ 'bear' \\
\hline
\end{tabular}

\subsection{Auditory analysis}

Auditory analysis was carried out in two phases: the first to determine if nasalisation occurs in non-nasal contexts, i.e. where no nasal is present, and if so, how it compares to nasal and pharyngeal contexts (including pharyngeals combined with nasal contexts); the second to determine what phonation types accompany the production of vowels in oral, nasal and pharyngeals environments. The first task resulted in a scaled judgment from 1 to 5 whereby 1 represented oral and 5 represented nasal. For the purpose of this study, the second phonation task was restricted to the following categorical judgment: breathy, modal, tense (the latter category included creaky, pharyngealised and laryngealised sub-categories). In order to minimize contextual influences (e.g. Beddor and Krakow, 1999; Kawasaki, 1986), the vowels were excised from their consonantal context; the extracted sound file was then windowed by a parabolic function to smooth the edges of the vowel, and the intensity was normalised to $65 \mathrm{~dB}$ so that the auditory judgments are not affected by the inherent intensity differences between the sounds. Each stimulus was sampled at $44 \mathrm{kHz}, 16$ Bit quantisation, monochannel. Tasks were run using Praat's experiment MFC as a rating test (Boersma and Weenink, 2017); each sound file was preceded and followed by half a second period of 
silence and could be played several times until an auditory category was chosen. The instructions for both tasks asked the participant to judge the nasality/voice quality of the stimuli on the presented scales.

The first author carried out the auditory analyses on the whole dataset and five additional raters (including the second author) carried out inter-rater reliability on $22 \%$ of the data. The $22 \%$ of the data comprised stimuli that were taken from all nine speakers and from all contexts. Given that there were restrictions in the combination of vowel and context, not all vowels were represented in all contexts. Thus, we aimed for words that had the three point vowels /i: a: u:/ where possible. In the end, each context was represented by one to three words, and 12 in the case of the isolation context, so that the subset is representative of the proportion of tokens per context in the main dataset. This allowed for a comparative judgment of the participants' productions from the five raters. The first additional rater (the second author) was a native speaker of Jordanian Arabic, while the remaining four were all native speakers of English (two British, one Irish and one Canadian) and were all phonetically trained with experience in phonation types. Prior to the beginning of the analysis the first author attended a three-day workshop on the perceptual judgement of voice quality by Janet Beck, an expert on Vocal Profile Analysis (Beck, 2005).

\subsection{Acoustic patterns applied in this study}

A host of measures were originally applied on the present data (see Alsiraih, 2013) but not all proved useful for this study due to a clash between what these measures can achieve in terms of distinguishing between nasalised and non-nasalised contexts on the one hand and pharyngealised and non-pharyngealised on the other. In the present study we concentrate on $A 1-P 0^{* 1}$ for low and low-mid vowels, $A 1-P 1^{*}$ for high and mid-high vowels (following Amino and Osanai, 2012, Chen, 1995, 1997, 2000; Chen et al., 2000; Chen et al., 2007), and

\footnotetext{
${ }^{1}$ The asterisk after $P 0, P 1$ and $H 1$ indicate normalised versions, see Section 6.5 below for more details.
} 
$B 1$ for measures of nasalisation, and $H 1^{*}-A 1$ for examining phonation types. Since $B 1$ can also index breathiness, the addition of the $H 1^{*}-A 1$ measure was made in order to allow a comparison of the patterns from both. If the $B 1$ and $H 1{ }^{*}-A 1$ patterns for pharyngeal consonants converge, then they would be taken as indexing breathiness rather than (or as well as) nasalisation in these consonants (Garellek, 2012; Kuang and Keating, 2012); however, if pharyngeals show different patterns in $B 1$ and $H 1^{*}-A 1$ then we might be able to disentangle the effects of breathiness and nasalisation.

The recordings were down-sampled to $10 \mathrm{kHz}$, low-pass filtered (to $5 \mathrm{kHz}$ ) and preemphasised with a factor of 0.98. Pre-emphasis was used to control for spectral tilt (Berger, 2007: 29). The target tokens were then manually segmented and a Praat script was used for the measurements detailed in this section, which were subsequently checked for errors. The script was developed by the second author (Al-Tamimi, J. and Khattab, 2011, 2015). Accordingly, at each portion (onset, midpoint and offset), a 40ms Kaiser 2 (Gaussian-like) window was generated, from which a DFT Spectrum was computed, following which a Logarithmic power spectral density, with a bin width of $11 \mathrm{~Hz}$ (using the LTAS function in Praat). The following acoustic events were first located at the onset, midpoint, and offset of the vowel (in order to investigate the degree and direction of influence of neighbouring consonants): $A 1, P 0, P 1, B 1$ and $H 1$. The automatic detection of $P 0$ and $P 1$ was not always successful, so we adopted a manual approach; we labelled $P 0$ as the highest peak located within the $200-500 \mathrm{~Hz}$ frequency range, and $P 1$ as the highest peak located between $F 1$ and $F 2$ in the $700-1300 \mathrm{~Hz}$ frequency range, as suggested in previous research (Chen, 1997: 2363). B1 was detected using Praat's standard detection algorithm, and visual inspection through LPC was carried out. $H 1$ was detected as being the highest peak in a range of frequencies defined as $f_{0}-\left(f_{0} / 10\right)$ for the lowest frequency and $f_{0}+\left(f_{0} / 10\right)$ for the highest $)$. The 
same applied to $A 1$, using the $F 1-B 1 / 2$ and $F 1+B 1 / 2$ ranges (Al-Tamimi, J., 2017; AlTamimi, J. and Khattab, 2011, 2015).

The acoustic measures above were then embedded in the form of relations, whereby the following behaviours were expected within a nasalised context: i) a decrease in the differences between the amplitude of the first formant and that of the extra nasal peaks (A1$P 0$ and $A 1-P 1)$, and ii) an increase in the bandwidth of the first formant $(B 1)$; in terms of phonation types, a lower value of $H 1-A 1$ was considered to denote a tense voice and a higher one a breathy voice (e.g. Chen, 2007; Keating and Esposito, 2007).

\subsection{Normalisation of nasalisation and phonatory measures}

Due to the boosting effects of $F 1$ and $F 2$ amplitude on $H 1, P 0$ and $P 1$, two normalisation techniques were employed. The first is based on the method described in Iseli et al (2007: 2285) as implemented in the Praat script (following Al-Tamimi, J., 2017; Al-Tamimi, J., and Khattab, 2011, 2015). This method uses formant frequencies, bandwidths and the sampling frequency to remove the boosting effects of surrounding formants. This enables the normalised amplitude of $H 1$ to be comparable to that of $H 1$ obtained through inversefiltering. The corrected value of each of $F 1$ and $F 2$ is then subtracted from that of $H 1$. The second method used the approximation techniques described by Chen (1995: 2452) to estimate the correction weights $\mathrm{T} 1$ and $\mathrm{T} 2$. Then the amplitudes of $P 0$ and $P 1$ were corrected for by subtracting each from the combined approximated amplitude values of both T1 and T2 respectively. Although these normalisation techniques in principle make it possible to compare low and high vowels across speakers and contexts, we followed the reviewers' recommendations and restricted the $A 1-P 0^{*}$ to the low and low-mid vowel contexts and $A 1$ $P 1 *$ to the high and mid-high ones. From this point onward, every time $P 0, P 1$ and $H 1$ are mentioned they will be followed by an asterisk, $P 0^{*}, P 1^{*}$ and $H 1^{*}$, denoting being corrected from the effect of $F 1$ and $F 2$. 


\subsection{Statistical Analysis}

Statistical analyses were run on auditory ratings and acoustic analyses using $\mathrm{R}$ (Microsoft $\mathrm{R}$ Open, version 3.4.0, R Core Team 2017). We started with statistical analyses on the auditory ratings. Inter-rater reliability (IRR) were computed on the ratings of the six raters (i.e., the five raters and the corresponding words obtained from the first author). Following the recommendations of Hallgren (2012), we used the Intra-Class Correlation (ICC) coefficient (Bartko, 1966). The ICCs are well suited to our data given that we have a fully crossed design where all raters judged the subset obtained from all participants. A two-way random agreement ICC was computed with the package irr (version 0.84, Gamer, Lemon, Fellows and Singh, 2012). Choosing both speakers and raters as random factors allows for generalisation of the results. The data from the six raters and each of the 45 words were used and ICC were obtained as averaged IRR for agreement on all data and per word level. The former allowed us to obtain an overall IRR score for the raters' performance while the latter allowed us to evaluate the word level variation with respect to the raters' judgments. IRR scores were then used to assess the degree to which the raters agreed in their decisions. Average ICC and confidence intervals are generated and will be used in the next section to evaluate the degree of IRR.

Following the IRR, we statistically analysed the data from each of the nasalisation and the voice quality ratings using Cumulative Logit Mixed Models, following Agresti (2002). Rating scales are inherently ordered, and this type of analysis allows for this ordered scale to be considered. This type of regression is a logistic regression with a specific link function, cumulative, and the resultant $\beta$ coefficients are proportional log odds (Agresti, 2002). We ran two types of Cumulative Logit Models: one with the inter-raters data (subset) and a second with the full data set from the first author (fullset) ( $\mathrm{R}$ script available at https://github.com/JalalAl-Tamimi/R-Rating-data). The aim was to evaluate whether the 
results between the two are comparable in the ratings of nasalisation and tense voice quality as judged by the six raters (on $22 \%$ of the data) and by the first author (on the full data set). To fit these Cumulative Logit Mixed Models, we used the function c $1 \mathrm{~mm}$ from the ordina 1 package (version 6.28; Christensen, 2015). The ratings (1 to 5 for nasalisation or 1 to 3 for voice quality), were the response part (as a factor), and the fixed effects were context (14 levels) and vowel (9 levels).

For both Full set and Subset models, we used various model specifications, e.g., random vs no-random effects; fixed effect interaction and simple effects, random slopes and random intercepts only. These various models were compared against each other using log Likelihood. The optimal model in both cases was the one with the context as a fixed effect and the following random structure for the full set: participant and item, and for the subset: participant, item and raters in addition to by-rater slopes for context. Vowels did not improve the model fit $(p>0.05)$

For the acoustic measures, namely $A 1-P 0^{*}$ (onset and offset) and $A 1-P 1^{*}$ (onset and offset), $B 1$ Mid, and $H 1^{*}-A 1$ (onset and offset),Linear Mixed Effects models were run with crossed random effect for subjects and items as implemented in the package 1 me4 (version 1.1-12, Bates, Maechler, Bolker, and Walker, 2015). For each acoustic correlate, an optimal model was obtained through model comparisons with Log Likelihood, and this was the one with context as a fixed factor and crossed random effects for participants and items. Vowels, either as simple effects or interactions, did not improve the model fit $(p>0.05)$. The intercept was the Isolation context taken to be the reference category.

For each model, we checked the normality of the random effects structures, and there were some minor deviations from normality in all cases, which is to be expected due to the variance level observed with respect to words and subjects. 
Finally, we ran an exploratory factor analysis as a dimensionality reduction technique to explore the relationships between the variables. Our aim was to evaluate the closeness of particular levels of the fixed factor (context) in order to shed light into whether pharyngeals (voiced or voiceless) are close to the nasal or to the oral contexts. Following this, we performed a Principal Component Analysis (PCA) using the function PCA from the package FactoMineR (version 1.36, Le, et al. 2008) and specified scale. unit = TRUE to allow for zscoring of all predictors using the correlation matrices. This z-scoring allows for "weak" predictors to be treated equally as "strong" predictors. The predictors were specified using the option quali.sup to allow the individual results to be assigned to a specific acoustic correlate. With the 14 levels of the context used as outcomes, we obtained 14 dimensions. Generally, the dimensions with individual variances above $5 \%$ are considered as the most important at explaining the data (Baayen, 2008, p. 121). The results reported in Section 7.3 showed that the first three Principal Components accounted for $79.2 \%$ of the cumulative variance. We will report on the percentage variance explained by the first dimensions, and will use a 3-dimensional space with the first three dimensions, generated using the package scatterp1ot3d (version 0.3-40, Ligges and Mächler, 2003).

\section{Results}

\subsection{Auditory impression of nasalisation and voice quality}

\subsubsection{Inter-Rater Reliability}

Inter-rater reliability (IRR) was obtained as an Intra-Class Correlation Coefficient (ICC) that assesses how the various speakers are judged as more or less nasal (or with tense vs breathy voice quality) across all raters. Following Hallgren (2012) and Cicchetti (1994), IRR is considered poor when ICC is less than 0.4 ; fair between 0.4 and 0.59 ; good between 0.6 and 0.74 and excellent between 0.75 and 1 ; ICC of 0 = random agreement; negative ICC equals systematic disagreement between raters. The averaged ICC agreement with crossed speaker 
and rater as random for the nasalisation judgment were at 0.74 (Confidence Intervals $(\mathrm{CI})$ : 0.52-0.92, $p<0.00001$ ), and 0.91 (CI: 0.80-0.97, $p<0.00001)$ for voice quality judgements. This high IRR is therefore considered good to excellent for nasalisation and excellent for voice quality. It should be noted that the ICC values are obtained for the overall subset data without any specificities for the words, i.e., this is the average of ICC agreement between all raters. Thus, we conducted a by-item IRR with the ICC being computed for each word in the subset. We used the same subset of the data, but specified ICC agreement computation between the six raters for each word.

Figure 4 shows the ICC scores for agreement for each word sorted from the lowest to the highest ICC value, with the CIs displayed, for both nasalisation (top) and voice quality (bottom). Starting with nasalisation, all but two items received ICC values above 0 , indicating various degrees of agreement across raters. Of these, more than half had their CIs on the positive side (see dotted vertical line), indicating reliable inter-rater agreement. The two nonsignificant items with negative ICCs were from the Isolation context. The overwhelming majority of items with relatively high ICC contained a nasal context (either at onset, offset or both), a pharyngeal (voiced or voiceless) or both. This indicates that raters had a relatively high level of agreement on these specific items. For those items with non-significant ICC (due to the CIS crossing the zero line), the majority contained oral-oral contexts and isolated vowels, with a few containing the pharyngeals (voiced or voiceless) in an oral context. The only item that is considered non-significant with a pharyngeal and a nasal context (item Se:n) had a relatively high ICC of 0.47 (CI: $-0.11-0.85, p=0.05)$. This result is interesting and suggests that nasal and pharyngeal (voiced and voiceless)+nasal ${ }^{2}$ contexts generated higher levels of agreement on their judgements than oral and pharyngeal+oral contexts, where raters

\footnotetext{
${ }^{2}$ Where '+' is used between two contexts, for example pharyngeal + oral, this is referring to both pharyngealoral and oral-pharyngeal contexts. Only where we are referring to a particular order do we use the dash instead (e.g. pharyngeal-oral)
} 
provided variable levels of agreements ranging from poor to fair IRR (with ICC between $0.12-0.48)$.

Moving on to the voice quality ICC per items, the results in Figure 4 (bottom) show that overall, all but 7 items received ICC values above 0 , indicating various degrees of agreement across raters. ICCs ranged between -0.69 (good level of disagreement) and 0.82 (excellent level of agreement). Once again, the non-significant ICCs (with CIs crossing the zero line and to the left of the dotted vertical line) are mainly from the isolation context (10 items out of 12), with four out of the six items with negative ICCs being from the isolation context. Compared with the nasalisation ratings, however, only 12 items had their CIs on the positive side (see dotted vertical line), indicating reliable inter-rater agreement. It should be noted that the high level of variation observed in these item judgments is consistent with the informal discussions we had with the four additional raters after the experiment had finished, who highlighted their difficulties in performing the voice quality rating experiment. Interestingly though, out of the 12 items receiving significantly positive ICCs (right of the dotted vertical line) nine contained a pharyngeal (voiced or voiceless) with an oral or nasal context. This suggests that raters had relatively high levels of agreements in their judgments of the voice quality associated with these items. 


\section{Agreement ICC for nasalisation}

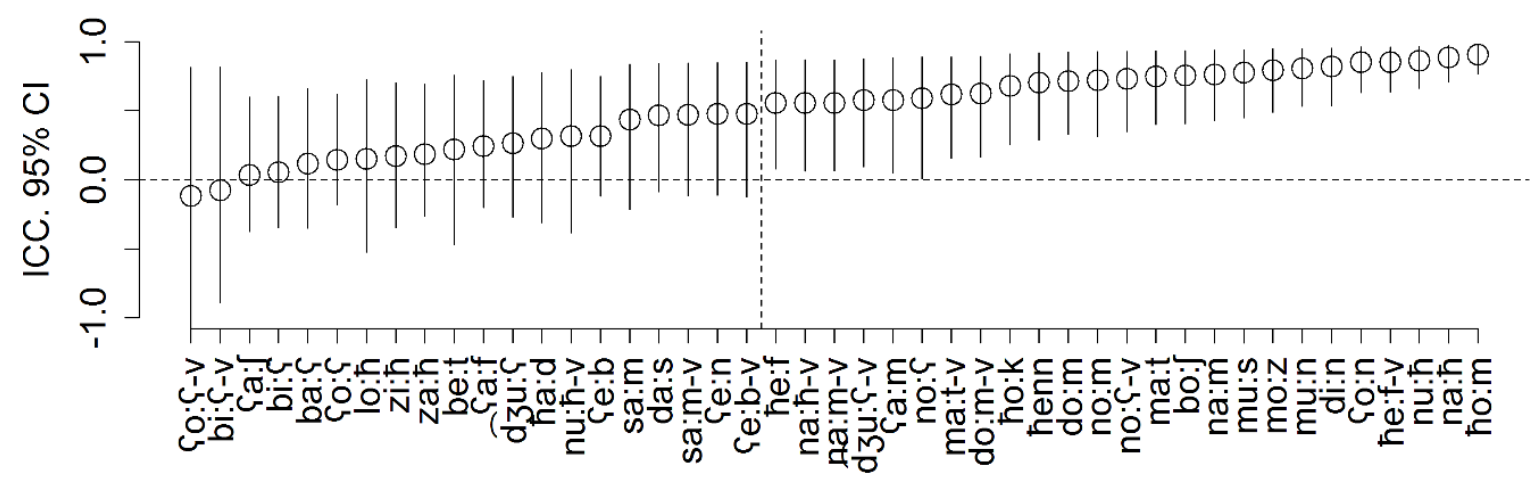

Agreement ICC for voice quality

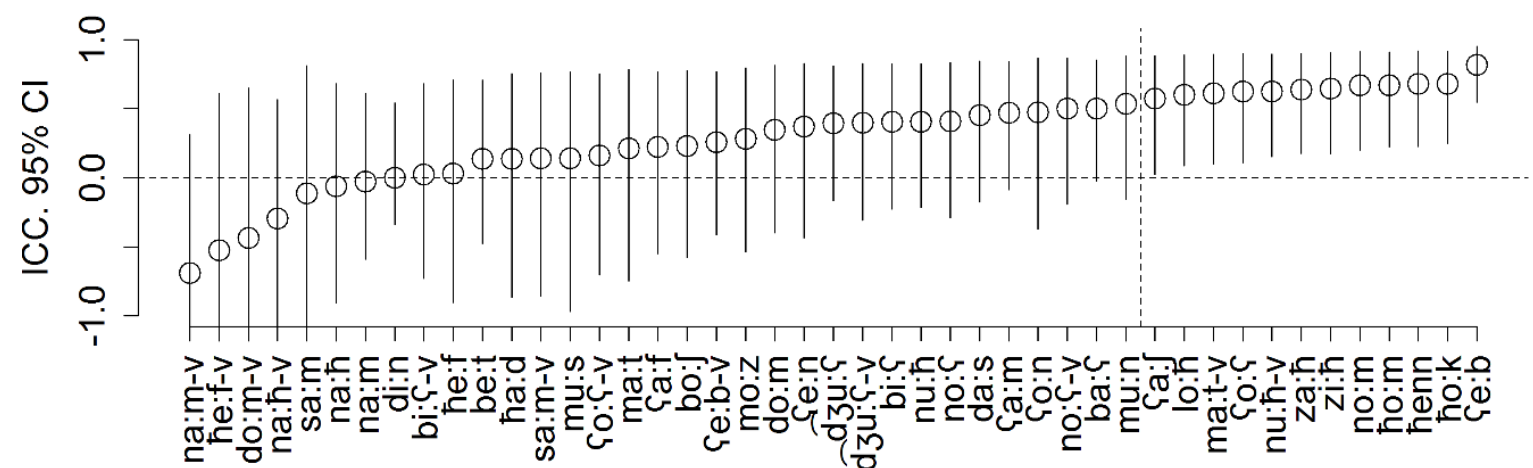

Figure 4: Intra-class correlation (ICC) scores for nasalisation (top) and voice quality (bottom) for each item used in the rating experiments (with confidence intervals, CI). Values below 0 are indicative of disagreements and those above show variable degrees of agreement (poor when ICC is less than 0.4; fair between 0.4 and 0.59 ; good between 0.6 and 0.74 and excellent between 0.75 and 1 ). Items to the right of the vertical dotted line indicate reliable agreement across all raters. Items ending with $-\mathrm{v}$ are indicative of the isolation context.

7.1.2. Cumulative Logit Mixed Effects Modelling

The results summarised above showed that overall, our six raters provided an overall high level of agreement when looking at their averaged ICC coefficients. The individual item analysis showed that there were specific items, mostly in the isolation context that had a poor to moderate levels of disagreement. However, these IRR scores do not allow us to show how the raters were performing on the specific contexts we are interested in, nor do they show how the full dataset is judged. Thus, we performed a Cumulative Logit Mixed Effects Modelling (CLMM) on the data from the six raters (on the subset of the data) and on the full dataset as judged by the first author. The aim here is to evaluate whether the judgements on the subset and the full dataset match in the magnitude and order, while taking into account the various types of variations observed in the data. This was done by considering speakers, 
items and raters (in the case of the subset) as random factors. This allows the CLMM to take into account this variation in reporting the results. The results of the CLMM from the subset and the full data set are summarised in Tables 2 and 3 (for nasalisation and voice quality respectively) and the predicted curves representing the overall change in judgments from 1 to 5 for nasalisation (or 1-3 for voice quality) for each specific context are presented in Figures 5 and 6 (for nasalisation and voice quality respectively). The results are shown for both the subset of the data (obtained from the six raters) and for the full dataset (from the first author).

CLMMs are proportional log odds models that evaluate the relationship between the fixed factor(s) on the overall increase in rating scales. The results summarised in the Tables 2 and 3 below show the $\beta$ coefficients (with the standard error, Wald's $\mathrm{z}$ and the $p$ values) for the 13 levels of the fixed factor (with Isolation being the reference value) and the $\beta$ coefficients (standard error, Wald's $\mathrm{z}$ ) for the combined effect of the thresholds of ratings. We choose Isolation as the reference level as we are taking this context to represent the control level, with no coarticulatory effects from surrounding environments. The interpretation of this proportional log odds model is that a unit increase in the linear predictor (context) is equal to a proportional change in the odds of the event (rating). To visualise the effects each of the 14 levels of the fixed effect (including Isolation), we used the predicted probabilities of each of the rating scales ( 5 in nasalisation and 3 in voice quality) that are based on the actual $\beta$ coefficients ordered from the lowest to the highest ${ }^{3}$.

Starting with nasalisation ratings, and through model comparisons, the results suggest that for the subset, a model with context as a fixed effect (with items, speakers and raters as random factors and by-raters random slopes for context) yielded an improvement to the model fit when compared to an intercept-only model $\left(\chi^{2}(13)=27.1, p<0.02\right)$. For the full set, a model with context as a fixed factor (and both items and speakers as crossed random

\footnotetext{
${ }^{3}$ These predicted curves and the figures were implemented in $\mathrm{R}$ via a modified version of the scripts available from http://web.maths.unsw.edu.au/ loicthibaut/ordinalLab.html and http://stats.stackexchange.com/questions/89474/interpretation-of-ordinal-logistic-regression.
} 
factors) yielded an improvement to the model fit when compared to an intercept-only model $\left(\chi^{2}(13)=214.87, p<0.0001\right)$. Table 2 shows the $\beta$ coefficients for the fixed factor and the threshold values of the CLMM, which are graphically presented as predicted curves for the five rating scales (Figure 5), for both the subset and the full dataset. In both cases, the threshold coefficients show negative coefficients for ratings 1-2, rising steadily to the maximum in 4-5, suggesting that the nasal ratings increase. The coefficients of each of the contexts, sorted from lowest to highest (in both Table 2 and Figure 5), show that all positive estimates are significantly and strongly associated with nasalisation ratings in both subset and full dataset.

An interesting pattern emerges in both the subset and full dataset as shown in Figure 5. In both datasets, there is a clear separation between two groups: in the first group, isolation, o-o and pharyngeals combined with orals attract mainly oral ratings (ratings of 1 and 2). In the second group, nasals and pharyngeals combined with nasals receive nasal ratings. In the full dataset only, when a nasal is associated with the voiced pharyngeal, i.e., n- $\mathrm{S}$, the rating of nasals (i.e., ratings of 4 and 5) is higher than that of the nasal associated with the oral context, i.e., n-o. This suggests that the addition of a pharyngeal to a nasal consonant may increase the percept of nasalisation, a pattern that is mirrored in some of the acoustic results as will be reported in Section 7.2.

Interestingly, all the contexts in both groups attracted ratings of 4 or 5 on the nasalisation scale (with varying proportions), suggesting that the presence of nasalisation is more gradual than categorical across contexts in IA and can be found even in oral environments, but to levels with lower than $20 \%$ ratings (for ratings of 3, 4 or 5). Moreover, in both subset and full dataset, voiced pharyngeals in nasal contexts yield higher nasal ratings than voiceless pharyngeals. But there are differences in some of the ratings between the two datasets: in the subset dataset, within nasal contexts (n-o and o-n), the presence of an initial 
nasal yields higher nasalisation ratings than a final nasal, but the pattern is reversed in the full dataset. Moreover, within oral contexts, an initial voiceless pharyngeal yields a relatively higher percept of nasalisation than the remaining oral contexts in the subset, but the lowest nasalisation ratings in the full dataset.

Overall, and for the purpose of this study, there were general agreements in both full and subset results on the nasalisation judgments of the oral contexts (including isolation, and pharyngeals in an oral context) and the nasal contexts (including pharyngeals in nasal contexts) as these formed clear separate groups along the nasalisation continuum in both data sets (Figure 5).

Table 2: Results of the Cumulative Logit Mixed model for the rating of nasalisation with the Fixed effect of the context sorted from lowest to highest $\beta$ coefficients and the thresholds for the 5 rating scales. The intercept is not presented in the table but shown in Figure 4.

\begin{tabular}{|c|c|c|c|c|}
\hline Fixed & $\beta$ & Std.Error & $\mathrm{z}$ value & $\operatorname{Pr}(>|z|)$ \\
\hline $0-\hbar$ & -1.1307 & 0.2536 & -4.459 & $8.23 \mathrm{E}-06$ \\
\hline O-O & -0.8159 & 0.197 & -4.142 & $3.44 \mathrm{E}-05$ \\
\hline h-o & -0.7144 & 0.2772 & -2.577 & 0.009964 \\
\hline S-O & -0.5713 & 0.2513 & -2.273 & 0.023027 \\
\hline o- $\mathrm{S}$ & -0.417 & 0.4007 & -1.041 & 0.298064 \\
\hline G-ৎ & 0.4066 & 0.773 & 0.526 & 0.598935 \\
\hline$n-\hbar$ & 2.1182 & 0.5719 & 3.704 & 0.000212 \\
\hline o-n & 2.7374 & 0.2153 & 12.712 & $\begin{array}{c}<2.00 \mathrm{E}- \\
16\end{array}$ \\
\hline S-n & 2.787 & 0.4627 & 6.023 & $1.71 \mathrm{E}-09$ \\
\hline h-n & 2.7956 & 0.7023 & 3.981 & $6.86 \mathrm{E}-05$ \\
\hline n-o & 2.9264 & 0.2805 & 10.434 & $\begin{array}{c}<2.00 \mathrm{E}- \\
16\end{array}$ \\
\hline$n-S$ & 3.0844 & 0.8325 & 3.705 & 0.000212 \\
\hline$n-n$ & 3.5382 & 0.3805 & 9.299 & $\begin{array}{c}<2.00 \mathrm{E}- \\
16\end{array}$ \\
\hline Threshold & Estimate & Std.Error & $\mathrm{z}$ value & \\
\hline $1 \mid 2$ & -1.1304 & 0.1941 & -5.825 & \\
\hline $2 \mid 3$ & 0.7217 & 0.1933 & 3.733 & \\
\hline \begin{tabular}{l|l|}
3 & 4 \\
\end{tabular} & 1.9543 & 0.1998 & 9.779 & \\
\hline $4 \mid 5$ & 3.513 & 0.2198 & 15.983 & \\
\hline
\end{tabular}



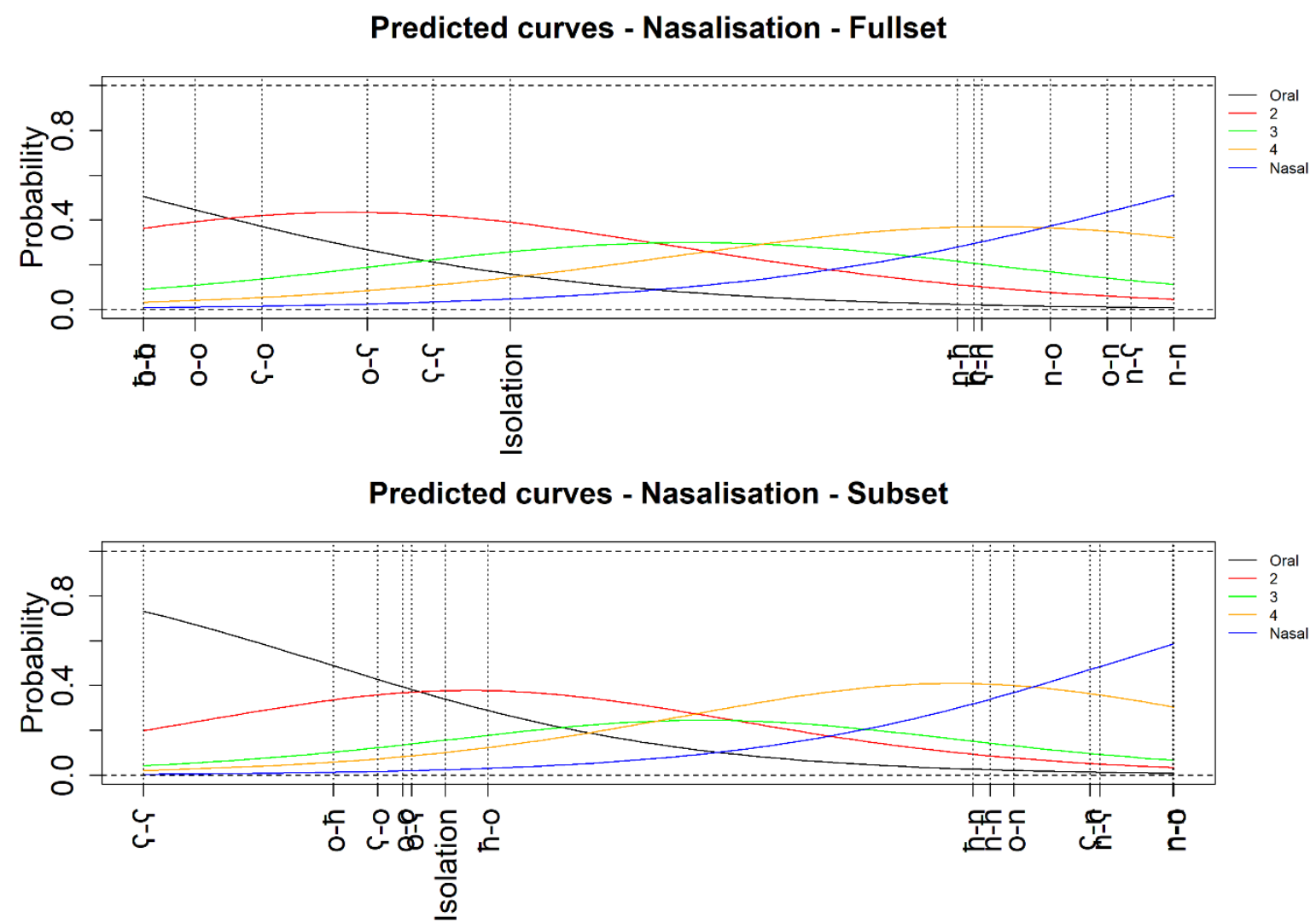

Figure 5: Cumulative predicted curves of nasalisation ratings. The y-axis shows probability from 0 to 1 ; the $x$-axis shows the contexts, sorted from lowest to highest contributor to nasalisation judgements. For instance, the blue line represents the nasal ratings rising steadily from approximately $0 \%$ in the 0 - $h$ category and reaching a maximum of $65 \%$ in the $\mathrm{S}-\mathrm{n}$ context.

Moving on to voice quality ratings, model comparisons showed that on the subset data, context yielded an improvement to the model fit $\left(\chi^{2}(13)=20.8, p=0.07\right)$; the same was obtained for the full dataset $\left(\chi^{2}(13)=278.8, p<0.0001\right)$. Table 3 shows the $\beta$ coefficient and the threshold values of the CLMM, which are graphically presented as predicted curves for the three judgement categories (Figure 6). In both the subset and the full dataset cases, the threshold coefficients show negative coefficients for categories breathy to modal (1-2), rising to a maximum in modal to tense (2-3). The coefficients of each of the contexts, sorted from lowest to highest, show that all contexts contribute to the tense rating in both subset and full dataset, with the context contributing to the highest rating being the $\mathrm{S}-\mathrm{S}$.

Table 3 and Figure 6 show that the majority of ratings on both the subset and the full dataset fall in the modal or tense categories, with very few breathy ratings overall, found 
mostly in isolation and nasal contexts. While nasal and oral (including isolation) contexts were mostly judged as modal ( $\sim 80 \%$ of the time), pharyngeal contexts were mainly judged as tense, especially voiced pharyngeal contexts (with the exception of n- $\uparrow$, which was judged as mainly modal in both subset and full datasets). The prevalence of a tense voice quality in Iraqi Arabic pharyngeals can potentially be due to their epilaryngeal constriction and stoplike manner of articulation, as described in the background section (also see Al-Siraih, 2013).

Table 3: Results of the Cumulative Logit Mixed model for the rating of VQ with the Fixed effect of the context sorted from lowest to highest $\beta$ coefficients and the thresholds for the 3 rating scales. The intercept is not presented in the table but shown in Figure 5.

\begin{tabular}{|c|c|c|c|c|}
\hline Fixed & $\beta$ & Std.Error & $\mathrm{z}$ value & $\operatorname{Pr}(>|z|)$ \\
\hline$n-n$ & 0.2507 & 0.4333 & 0.579 & 0.5629 \\
\hline n-o & 0.3253 & 0.3088 & 1.053 & 0.2921 \\
\hline o-n & 0.4821 & 0.2422 & 1.991 & 0.0465 \\
\hline$n-\subseteq$ & 0.5253 & 1.0527 & 0.499 & 0.6178 \\
\hline $\mathrm{O}-\mathrm{O}$ & 1.6481 & 0.2414 & 6.827 & $8.67 \mathrm{E}-12$ \\
\hline h-n & 1.9123 & 0.7562 & 2.529 & 0.0114 \\
\hline $0-\hbar$ & 3.3376 & 0.2929 & 11.396 & $\begin{array}{c}<2.00 \mathrm{E}- \\
16\end{array}$ \\
\hline$n-\hbar$ & 3.6287 & 0.6157 & 5.893 & 3.79E-09 \\
\hline h-o & 3.7814 & 0.2741 & 13.794 & $\begin{array}{c}<2.00 \mathrm{E}- \\
16\end{array}$ \\
\hline o- & 4.1669 & 0.4484 & 9.293 & $\begin{array}{c}<2.00 \mathrm{E}- \\
16\end{array}$ \\
\hline S-n & 4.3397 & 0.4286 & 10.126 & $\begin{array}{c}<2.00 \mathrm{E}- \\
16\end{array}$ \\
\hline S-O & 5.0488 & 0.3068 & 16.456 & $\begin{array}{c}<2.00 \mathrm{E}- \\
16\end{array}$ \\
\hline S-S & 5.7095 & 1.1954 & 4.776 & $1.79 \mathrm{E}-06$ \\
\hline Threshold & Estimate & Std.Error & $\mathrm{z}$ value & \\
\hline $1 \mid 2$ & -1.543 & 0.1859 & -8.3 & \\
\hline $2 \mid 3$ & 3.9163 & 0.239 & 16.39 & \\
\hline
\end{tabular}




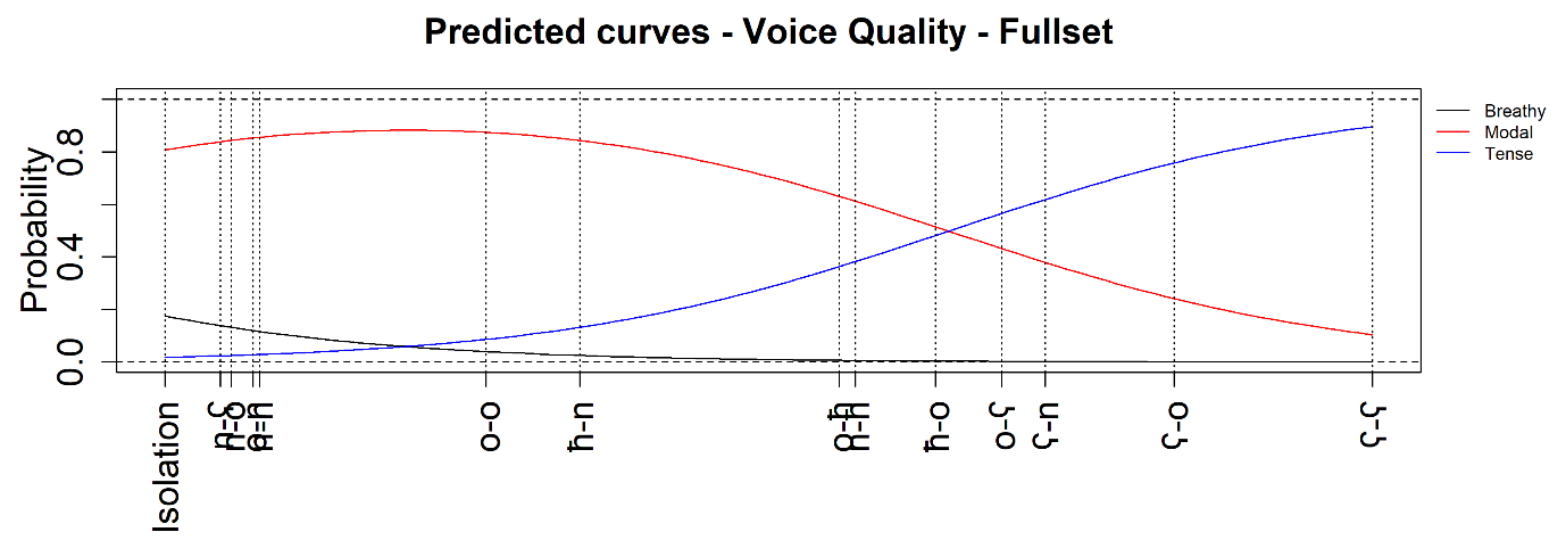

Predicted curves - Voice Quality - Subset

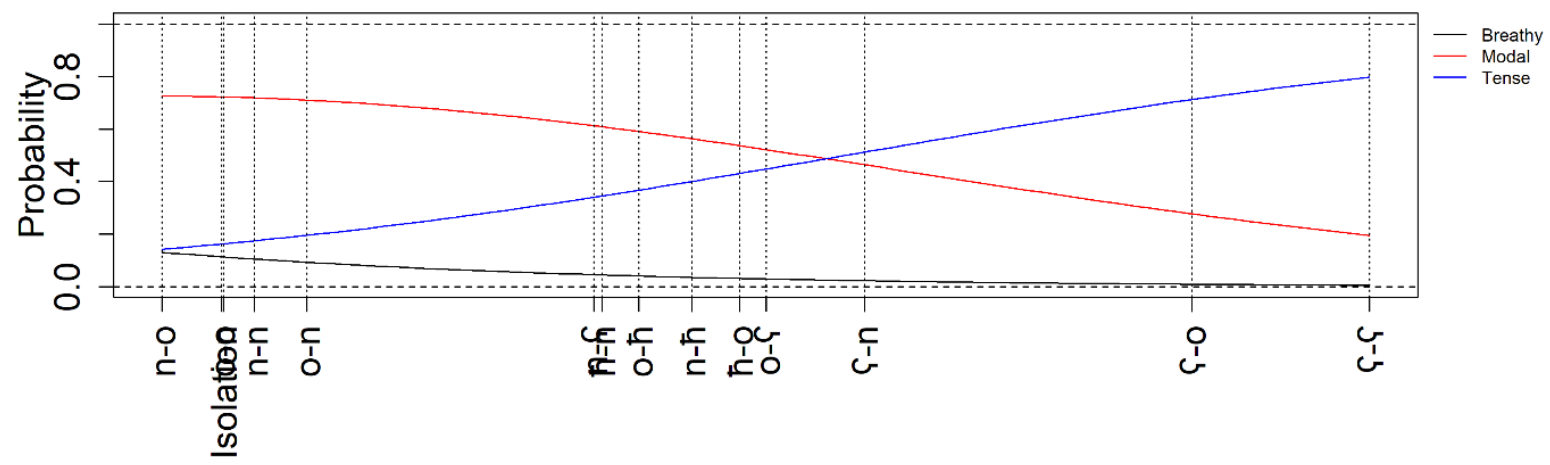

Figure 6: Cumulative predicted curves of voice quality judgements. The $y$-axis shows probability from 0 to 1; the $x$-axis shows the contexts, sorted from lowest to highest contributor to VQ judgements.

Overall, results of the auditory analyses on the subset (using the six raters) and the full dataset clearly separated oral and pharyngeal+oral contexts on the one hand, and nasal and pharyngeal+nasal contexts on the other. There was an increased percept of nasalisation of pharyngeals in a nasal context compared to when they are associated with an oral context. Importantly though, the results suggest that pharyngeals in a nasal context elicit a nasalisation percept that is comparable to that of oral+nasal environments, with only the voiced pharyngeal in the $n-\uparrow$ context showing potential for added nasalisation.

In addition, pharyngeals triggered a percept of tense voice quality. In the next section, we will be looking at the acoustic correlates of nasalisation, e.g., $A 1-P 0^{*}, A 1-P 1^{*}$ and onset and offset and $B 1$ Mid in order to shed light on the effects pharyngeals have on these metrics when associated with nasals. Then we present the results of the voice quality correlate in 
order to evaluate any decrease in $\mathrm{H} 1^{*}$-A 1 in the pharyngeal context that is taken to be an indication of tense voice quality.

\subsection{Acoustic results}

Here and elsewhere the values resulting from the $A 1-P 0^{*}$ and $A 1-P 1^{*}$ measurements are interpreted as follows (see Chen 1995, 1997, 2000; Chen et al. 2000, 2007; and Berger 2007): the lower the amplitude difference the more nasalisation the context is deemed to exhibit, with phonological nasal contexts expected to have the lowest of all values, the oral and isolated vowel contexts the highest, and the pharyngeal and pharyngeal-nasal contexts as the experimental ones. The following sections will present results at onset and offset portions only in order to save space and due to the fact that we are mainly interested in the influence of the pharyngeals on vowel edges. When presenting results at onset, we mainly discuss the effect of the initial consonants (although the tables and figures show all contexts); similarly, when we present results at offset, we mainly focus on the effect of the final consonants. The results for this section will be presented in three ways. First, through model comparisons, we report on whether context improves the model through log likelihood comparison. Next, we report on the results of the Linear Mixed Effects models in Tables 4 to 7 . The $p$ values in these tables were obtained using the package 1merTest (version 2.0-32, Kuznetsova, Bruun Brockhoff and Haubo Bojesen Christensen, 2016). The third statistical report was through multiple pairwise comparisons using the package 1 smeans (version 2.26-3, Lenth, 2016). This multiple comparison allows us to evaluate the differences between specific levels of the fixed factor. We graphically present the results of the predicted values from each of the acoustic correlates for each of the 14 levels of contexts, based on the fitted model. These were generated using ggplot2 (version 2.2.1, Wickham, 2009). We used boxplot to show the within category variation and a smoothed curve to display the trend in the effect of each level of context on the acoustic results. 


\subsubsection{A1-P0*}

As a reminder, this measure was used for low and lower-mid vowels only. In onset position, context showed an improvement to model fit when compared to an intercept only model $\left(\chi^{2}(13)=69.1, p<0.0001\right)$. Specifically, the isolation and oral contexts pattern together and show the typical high amplitude values expected for oral contexts, in line with Chen's $10 \mathrm{~dB}$ cut off suggestion (Table 4 and Figure 7). Nasals in onset position (n-o and n-n) show values being below the $10 \mathrm{~dB}$ range, with $\mathrm{n}$-o having significantly lower values than isolation contexts (pairwise comparisons, $p<0.001$ ) and $n-n$ approaching significance with a $3.87 \mathrm{~dB}$ difference $(p=0.07)$. When pharyngeals are in onset position and combined with oral contexts, they either show an increase in $A 1-P 0^{*}$ compared to isolation ( $\hbar-0, \mathrm{G}-\mathrm{o}$ ) or comparable values ( $(\mathcal{\complement}-\mathcal{\complement})$, suggesting no increased nasalisation is associated with pharyngeals on their own. The same applies when comparing $\hbar-o$ and $\mathrm{S}-\mathrm{o}$ with o-o (pairwise comparisons, $p<0.0001$ for both). And while we are looking at $A 1-P 0^{*}$ at onset, it is worth noting that o- $\hbar$ and o- $\subseteq$ also show subtle non-significant increases in amplitude compared with o-o (Figure 7).

In nasal-pharyngeal contexts, $\mathrm{n}-\Upsilon_{\text {shows }}$ the lowest $A 1-P 0 *$ values, with an average $3 \mathrm{~dB}$ difference with $n-n$ and $2 \mathrm{~dB}$ with n-o. While the difference between $\mathrm{n}-\mathrm{S}$ and these other onset nasal contexts is not significant, it is worth noting that $A 1-P 0^{*}$ values for o- $\complement$ and $\mathrm{n}-\complement$ show a larger amplitude drop $(7.5 \mathrm{~dB}, p=0.079)$ than those between o-o and $\mathrm{n}-\mathrm{o}(1.77 \mathrm{~dB}$, nonsignificant), suggesting a role for $\subseteq$ in an increased effect of nasalisation, which is in line with the auditory impression of this context. The relatively high amplitude values for the voiced pharyngeal in an oral context is met by a large drop in the $n-\uparrow$ context, potentially suggesting different velopharyngeal configurations for the voiced pharyngeal in oral and nasal contexts, an issue we come back to in the discussion. A1- $P 0^{*}$ values for $\mathrm{n}-\mathrm{h}$ also do not show a 
significant difference with either oral contexts (isolation, o-o or o- $\hbar$ ) or nasal ones (n-n, n-o, h-n).

Moving on to pharyngeals in a nasal context, $\hbar-n$ does not differ significantly from oral contexts (isolation, o-o or h-o) or nasal ones (o-n or n-n). A1-P0* values for $\mathrm{G}-\mathrm{n}$ do not differ significantly from isolation, and in fact they are significantly higher than for o-o (pairwise comparison, $p<0.01$ ), showing the big effect of the pharyngeal on this onset measure. $\mathrm{G}-\mathrm{n}$ also

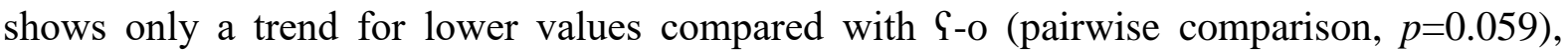
suggesting a reduced anticipatory effect of nasalisation compared with the perseverative effect in $\mathrm{n}-\mathrm{\complement}$. Note, however, that $\mathrm{\complement}$-o showed the highest mean $A 1-P 0 *$ values across all contexts, making it hard to see an effect of a final nasal in this onset measure.

In offset position, model comparison showed that the inclusion of context as a fixed factor did not improve model fit compared with an intercept-only model $\left(\chi^{2}(13)=21.4\right.$, $p=0.06)$. This suggests that any changes observed in consonantal contexts have no association with $A 1-P 0 *$ differences (Table 4 and Figure 7 ). This is an interesting finding, potentially showing a stronger influence of nasals (and nasals+pharyngeals) at onset rather than offset. We still examined whether any contexts showed statistical differences, and only two differed from isolation, namely o- $\hbar$ and o- $\mathrm{G}$, which showed an increase in $A 1-P 0^{*}$ by 5 to $14 \mathrm{~dB}$. This once again shows the very high amplitude for pharyngeals in oral contexts. This means that, while $\mathrm{n}-\hbar$ and $\mathrm{n}-\mathcal{Y}$ do not show significantly different $A 1-P 0^{*}$ values from other nasal contexts (n-n, n-o or o-n), the drop from o- $\hbar$ to $n-\hbar$ is larger than from o-o to n-o ( $5 d B$ vs $2 d B)$, and the drop from o- $\subseteq$ to $n-\uparrow$ is even higher $(16 \mathrm{~dB})$. Although non-statistically significant (with $n-0$ showing marginal significance level), nasal contexts generally had lower $A 1-P 0^{*}$ values than oral and isolation contexts, in line with what was found at onset. The $3 \mathrm{~dB}$ difference as observed in n-n, n-o, h-n and $\mathrm{S}-\mathrm{n}$ is beyond the threshold for amplitude discrimination, as reported in Stevens (1998). 
Table 4: Results of the Linear Mixed Models for $A 1-P 0^{*}$ at the onset and offset of the vowel in the 13 levels of the fixed effect context. The Intercept is the Isolation context and the $\beta$ coefficients represent the difference between the Intercept and the actual value for the level of the fixed effect. The $p$ values are generated using 7 merTest.

\begin{tabular}{|c|c|c|c|c|c|c|c|c|}
\hline & \multicolumn{4}{|c|}{ Onset } & \multicolumn{4}{|c|}{ Offset } \\
\hline & $\beta$ & Std.Error & t value & $\operatorname{Pr}(>|t|)$ & $\beta$ & Std.Error & t value & $\operatorname{Pr}(>|t|)$ \\
\hline (Intercept) & 12.6281 & 1.1504 & 10.977 & 9.19E-07 & 12.8172 & 1.3931 & 9.2 & $1.69 \mathrm{E}-07$ \\
\hline O-O & -2.9172 & 0.7747 & -3.766 & 0.000188 & 0.9372 & 1.7128 & 0.547 & 0.5847 \\
\hline o- $\hbar$ & -1.5455 & 1.0136 & -1.525 & 0.12806 & 5.2023 & 2.2361 & 2.326 & 0.0208 \\
\hline o- & -1.588 & 2.853 & -0.557 & 0.578051 & 14.1527 & 6.3144 & 4.27 & 0.0002 \\
\hline h-o & 1.8612 & 0.8015 & 2.322 & 0.020887 & 0.97 & 1.7542 & 0.553 & 0.581 \\
\hline S-O & 4.2881 & 1.0735 & 3.995 & $7.78 \mathrm{E}-05$ & 1.1761 & 2.3628 & 0.498 & 0.6191 \\
\hline $\mathrm{S}-\mathrm{S}$ & 1.1609 & 2.853 & 0.407 & 0.684246 & -6.9755 & 3.3144 & -2.105 & 0.027 \\
\hline o-n & -2.9257 & 0.8695 & -3.365 & 0.00081 & -1.1512 & 1.9408 & -0.593 & 0.5534 \\
\hline n-o & -4.5076 & 1.2057 & -3.739 & 0.000207 & -3.3503 & 1.6686 & -2.007 & 0.021 \\
\hline$n-\hbar$ & -1.3805 & 2.853 & -0.484 & 0.628681 & -0.1833 & 6.3144 & -0.029 & 0.9769 \\
\hline$n-\Upsilon$ & -6.4265 & 2.853 & -2.253 & 0.024716 & -1.5134 & 6.3144 & -0.24 & 0.8108 \\
\hline h-n & -0.3474 & 2.094 & -0.166 & 0.868346 & -2.4189 & 4.5682 & -0.53 & 0.5972 \\
\hline S-n & 1.2086 & 1.3384 & 0.903 & 0.367138 & -3.3675 & 2.9364 & -1.147 & 0.2529 \\
\hline$n-n$ & -4.4513 & 2.0263 & -2.197 & 0.028469 & -3.6598 & 4.4898 & -0.815 & 0.4157 \\
\hline
\end{tabular}




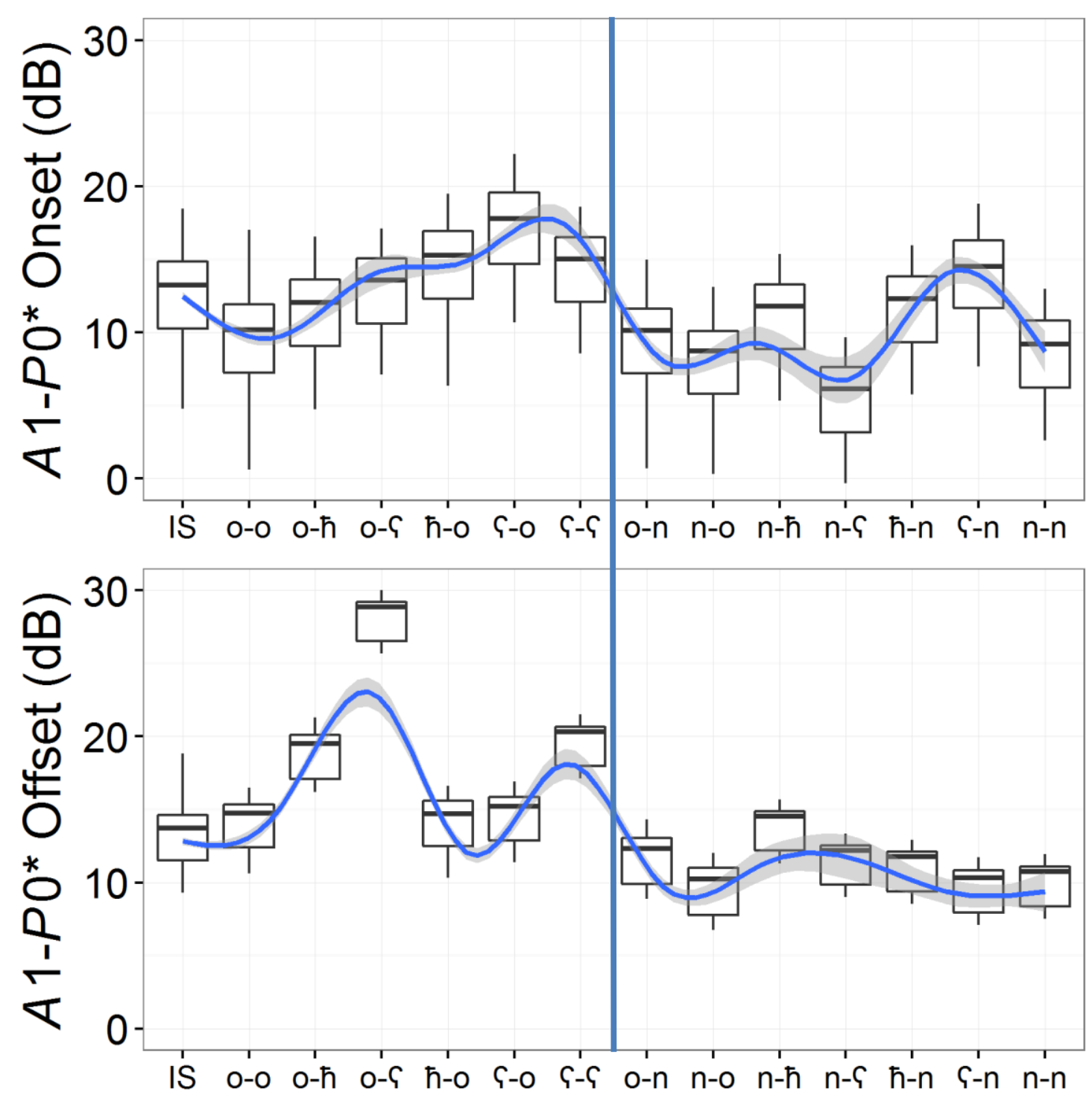

Figure 7: $A 1-P 0 *$ values within oral (left) and nasal (right) contexts at onset (top) and offset (bottom).

\subsubsection{A1-P1*}

In onset position, context showed an improvement to model fit when compared to an intercept only model $\left(\chi^{2}(8)=22.6, p<0.005\right)$. It should be noted that only nine contexts were used here rather than the full 14 due to restrictions in the analyses to only high and mid-high vowel contexts. The oral context, when compared to isolation, showed no significant differences and the mean values of $A 1-P 1 *$ were close to $18 \mathrm{~dB}$ (Table 5 and Figure 8 ). Nasals in onset position (n-o and n-n) show significantly lower values than isolation contexts $(p<0.05)$, by 3 to $5 \mathrm{~dB}$, however the values are still beyond the $10 \mathrm{~dB}$ threshold. When the voiced pharyngeal is in onset position and combined with oral contexts ( $\mathrm{S}-\mathrm{O})$, it showed a 
decrease in $A 1-P 1^{*}$ compared to isolation $(p<0.05)$, which mirrors what is observed in the nasal only contexts here. The voiceless pharyngeal shows a similar trend, but the difference between $0-\hbar$ and isolation is not significant. Interestingly, when the voiced pharyngeal is in final position and associated with an oral environment (o-ৎ), it shows the same increase over the o-o and isolation contexts that was found for $A 1-P 0^{*}$, with between 3 and $4 \mathrm{~dB}$ difference. In nasal contexts, only the voiceless nasal-pharyngeal context is available for the high vowels; n- $\hbar$ shows the lowest $A 1-P 1 *$ values (by $6 \mathrm{~dB}$ ), but is not statistically different from n-n or n-o.

In offset position, context showed an improvement to model fit when compared to an intercept only model $\left(\chi^{2}(8)=16.8, p<0.05\right)$. More specifically, the oral context, when compared to isolation, showed no significant differences and the mean values of $A 1-P 1^{*}$ were close to the $15 \mathrm{~dB}$ (Table 5 and Figure 8). Nasals in offset position (o-n and n-n) show significantly lower values than isolation contexts, by 3 to $6 \mathrm{~dB}$. It is only in $n-n$ context that the mean $A 1-P 1^{*}$ at offset is below the $10 \mathrm{~dB}$. When pharyngeals are in final position and combined with orals, they show a significant decrease in $A 1-P 1^{*}$ by 3 to $5 \mathrm{~dB}$, with o- $\hbar$ reaching the $10 \mathrm{~dB}$ threshold but not crossing it. This decrease may be indicative of a slight nasalisation in this context although this is not comparable to what is seen in the n-n context. The only pharyngeal-nasal context, $n-\hbar$, is not statistically different from isolation.

In sum, although this measure is successful at separating isolation/oral from $n-n$ contexts, there is only a weak trend for pharyngeals having lower $A 1-P 1^{*}$ than oral or isolation contexts, but it is significant for $\mathrm{S}_{-\mathrm{o}}$ in onset and o- $\mathrm{h}$ in offset. The lack of a voiced pharyngeal in the nasal contexts and the pre-dominance of high vowel tokens for this measure may make it harder to provide stronger evidence for pharyngeals being accompanied with nasalisation. 
Table 5: Results of the Linear Mixed Models for $A 1-P 1^{*}$ at the onset and offset of the vowel in the 13 levels of the fixed effect context. The Intercept is the Isolation context and the $\beta$ coefficients represent the difference between the Intercept and the actual value for the level of the fixed effect. The $p$ values are generated using 7merTest.

\begin{tabular}{|c|c|c|c|c|c|c|c|c|}
\hline & \multicolumn{4}{|c|}{ Onset } & \multicolumn{4}{c|}{ Offset } \\
\cline { 2 - 9 } & $\beta$ & $\begin{array}{c}\text { Std.Erro } \\
\mathrm{r}\end{array}$ & $\begin{array}{c}\mathrm{t} \\
\text { value }\end{array}$ & $\operatorname{Pr}(>|\mathrm{t}|)$ & $\beta$ & $\begin{array}{c}\text { Std.Erro } \\
\mathrm{r}\end{array}$ & $\begin{array}{c}\mathrm{t} \\
\text { value }\end{array}$ & $\operatorname{Pr}(>|\mathrm{t}|)$ \\
\hline $\begin{array}{c}\text { (Intercept } \\
\text { ) }\end{array}$ & 17.954 & 0.9377 & $\begin{array}{c}19.14 \\
6\end{array}$ & $6.66 \mathrm{E}-16$ & 15.664 & 1.347 & $\begin{array}{c}11.62 \\
6\end{array}$ & $4.92 \mathrm{E}-09$ \\
\hline $\mathrm{o}-\mathrm{o}$ & -0.103 & 1.272 & -0.081 & 0.93553 & -0.515 & 1.611 & -0.32 & 0.74951 \\
\hline $\mathrm{o}-\mathrm{h}$ & -4.6712 & 1.7032 & -2.743 & 0.00646 & -5.567 & 2.164 & -2.573 & 0.01071 \\
\hline $\mathrm{o}-\mathrm{C}$ & 3.5645 & 1.2477 & 2.857 & 0.00874 & -3.026 & 1.387 & -2.182 & 0.02056 \\
\hline $\mathrm{S}-\mathrm{o}$ & -4.4656 & 1.4347 & -3.112 & 0.00205 & 1.134 & 1.817 & 0.624 & 0.53316 \\
\hline $\mathrm{o}-\mathrm{n}$ & -1.8126 & 1.3214 & -1.372 & 0.17113 & -3.619 & 1.684 & -2.149 & 0.03255 \\
\hline $\mathrm{n}-\mathrm{o}$ & -3.5462 & 1.5158 & -2.34 & 0.01988 & -1.85 & 1.937 & -0.955 & 0.34041 \\
\hline $\mathrm{n}-\mathrm{h}$ & -6.1449 & 2.1974 & -2.796 & 0.00971 & -1.091 & 4.11 & -0.265 & 0.79085 \\
\hline $\mathrm{n}-\mathrm{n}$ & -5.2917 & 1.7032 & -3.107 & 0.00207 & -6.3 & 2.164 & -2.911 & 0.00395 \\
\hline
\end{tabular}




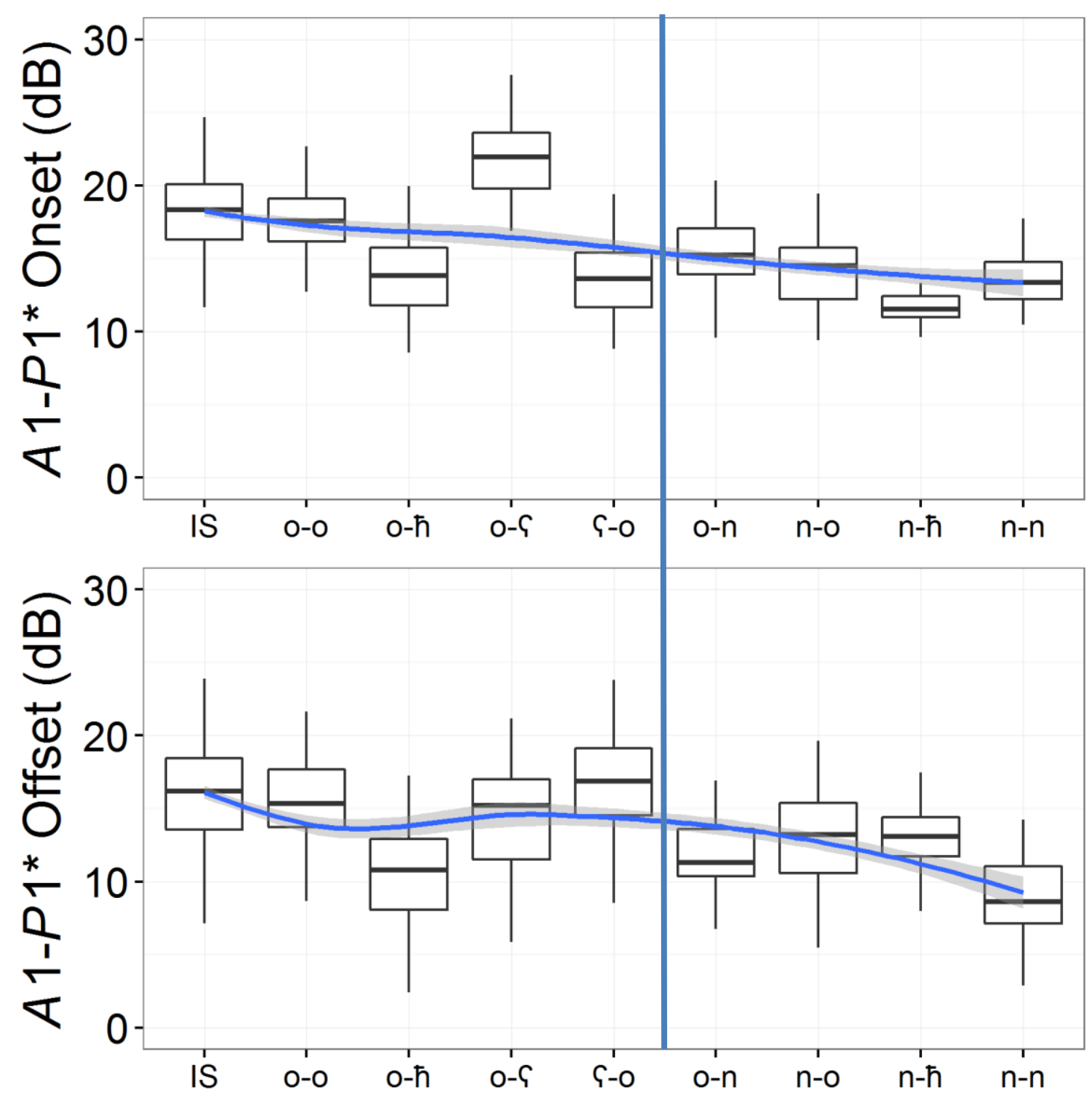

Figure 8: $A 1-P 1 *$ values within oral (left) and nasal (right) contexts at onset (top) and offset (bottom).

\subsubsection{First formant Bandwidth (B1)}

As a reminder, this first formant bandwidth measure is expected to show an increase in frequency in a nasalised context (Beddor, 1983; Hawkins and Stevens, 1985; Klatt and Klatt, 1990; Trittin and de Santos y Lléo, 1995; Chen et al., 2007; Beddor, 2007; Berger, 2007). The criteria used in the literature to differentiate vowels with nasalisation from those with no nasalisation suggest a $B 1$ measure between $200 \mathrm{~Hz}$ and $300 \mathrm{~Hz}$ for the former and below $200 \mathrm{~Hz}$ for the latter. As noted for the $A 1-P 0^{*}$ and $A 1-P 1^{*}$ measure, it is important to point out that this measure is being applied in this study on words containing a variety of vowels 
and consonants not found in other investigated languages; therefore, the $B 1$ values need to be evaluated with care as they might show combined effects of nasal-oral environments alongside different vowel and consonant qualities. Only measures at midpoint are presented here because the measures at onset are very similar while those at offset show less distinction between nasal and non-nasal contexts.

When running statistical analyses on $B 1$, and when compared with an intercept-only model, context showed an improvement to the model fit $\left(\chi^{2}(13)=96.2, p<0.0001\right)$. On the whole, $B 1$ values are higher in nasal than non-nasal contexts, with a steady increase from the isolation and oral contexts to nasals. (Table 6 and Figure 9), Moreover, the cut-off point between nasal and non-nasal environments seems to be closer to a $140 \mathrm{~Hz}$ rather than the $200 \mathrm{~Hz}$ reported elsewhere. It should be noted that there is some variation as observed in Figure 9 in all categories due to differences between subjects and items. The oral context shows a tendency for a lower $B 1$ than isolation, but this is still within the $100 \mathrm{~Hz}$ bandwidth.

Moving on to the nasal only contexts, (i.e., n-o, o-n and n-n), the results show a significant increase from 40 to $80 \mathrm{~Hz}$ in the nasal contexts compared with Isolation (Table 6). Interestingly, pharyngeals combined with oral contexts (i.e., o- $\hbar, \hbar-o, o-\varsigma, \varsigma-\varsigma$, but not $९-0$ ) also show a marginal increase in $B 1$ compared with isolation, but this is only significant for

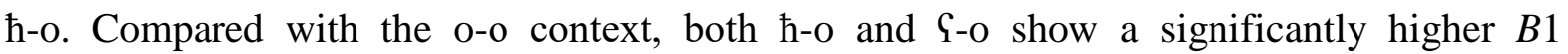
(pairwise comparison, $p<0.001$ and $p<0.05$ respectively). This is the first result that shows pharyngeal-oral contexts as being significantly different from other oral contexts, but their frequencies were also significantly lower than for nasal contexts (pairwise comparisons, $p<0.05$ for $\hbar-\mathrm{o} v s \mathrm{n}-\mathrm{o}$ and $\hbar-\mathrm{o} v s \hbar-\mathrm{n} ; p<0.01$ for $\mathrm{o}-\hbar v s$ o-n but $\mathrm{o}-\hbar$ not significantly different

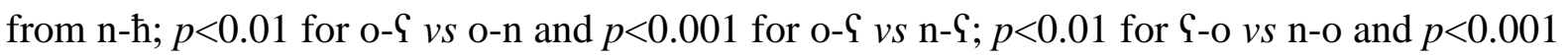
G-o vs $\mathrm{G}-\mathrm{n})$. This suggests more gradation in the potential presence of nasalisation across the 
four main contexts (oral/isolation, pharyngeal+oral, pharyngeal+nasal, and nasal). $B 1$ is also the only measure which shows potential nasalisation in both $\hbar$ and .

When pharyngeals are combined with nasals, they show an increase in $B 1$ frequency that is comparable to the nasal contexts, especially for $\hbar-n, n-\varsigma$, and $\uparrow-n ; n-\hbar$, which all have significantly higher $B 1$ frequency than isolation. The highest $B 1$ frequency was found in the $\mathrm{n}-\mathrm{\complement}$ context, which was on average $140 \mathrm{~Hz}$ when compared to isolation. This is interesting, as it seems that this context strongly affects the vowel to levels not seen for nasals on their own, and can potentially be claimed to be a "true" nasal environment as per thresholds used in the literature, with $B 1$ ranging between 200 and $300 \mathrm{~Hz}$ (see background). $B 1$ frequency for $\mathrm{n}-\mathrm{S}$ was in fact significantly higher than for $\mathrm{n}-\mathrm{o}$ (pairwise comparison, $p<0.01$ ), and between 40 and $52 \mathrm{~Hz}$ higher than $\mathrm{n}-\mathrm{o}$ and $\mathrm{n}-\mathrm{n}$, though not significantly different. Further, $\mathrm{S}-\mathrm{n}$ had significantly higher $B 1$ frequency than o-n (pairwise comparison, $p<0.01$ ), and mean $B 1$ for h-n was $40 \mathrm{~Hz}$ higher than o-n but failed to reach significance.

Table 6: Results of the Linear Mixed Models for B1 at the midpoint of the vowel in the 13 levels of the fixed effect context. The Intercept is the Isolation context and the $\beta$ coefficients represent the difference between the Intercept and the actual value for the level of the fixed effect. The $p$ values are generated using 7 merTest.

\begin{tabular}{|c|c|c|c|c|}
\hline & $\beta$ & Std.Error & t value & $\operatorname{Pr}(>|t|)$ \\
\hline (Intercept) & 104.785 & 12.876 & 8.138 & $1.51 \mathrm{E}-05$ \\
\hline O-O & -14.979 & 7.901 & -1.896 & 0.05846 \\
\hline $0-\hbar$ & 1.668 & 10.413 & 0.16 & 0.87277 \\
\hline o- & -17.841 & 19.157 & -0.931 & 0.35198 \\
\hline h-o & 20.807 & 9.418 & 2.209 & 0.02779 \\
\hline S-O & 9.374 & 10.109 & 0.927 & 0.35422 \\
\hline S-S & 14.754 & 35.623 & 0.414 & 0.67889 \\
\hline o-n & 40.505 & 8.744 & 4.632 & $4.17 \mathrm{E}-06$ \\
\hline n-o & 51.045 & 11.617 & 4.394 & $1.30 \mathrm{E}-05$ \\
\hline$n-\hbar$ & 10.368 & 26.668 & 0.389 & 0.69757 \\
\hline$n-\mathrm{C}^{2}$ & 141.172 & 34.129 & 4.136 & $4.08 \mathrm{E}-05$ \\
\hline$\hbar-n$ & 87.039 & 26.02 & 3.345 & 0.00091 \\
\hline S-n & 88.838 & 16.489 & 5.388 & $1.14 \mathrm{E}-07$ \\
\hline$n-n$ & 85.942 & 15.189 & 5.658 & $2.36 \mathrm{E}-08$ \\
\hline
\end{tabular}




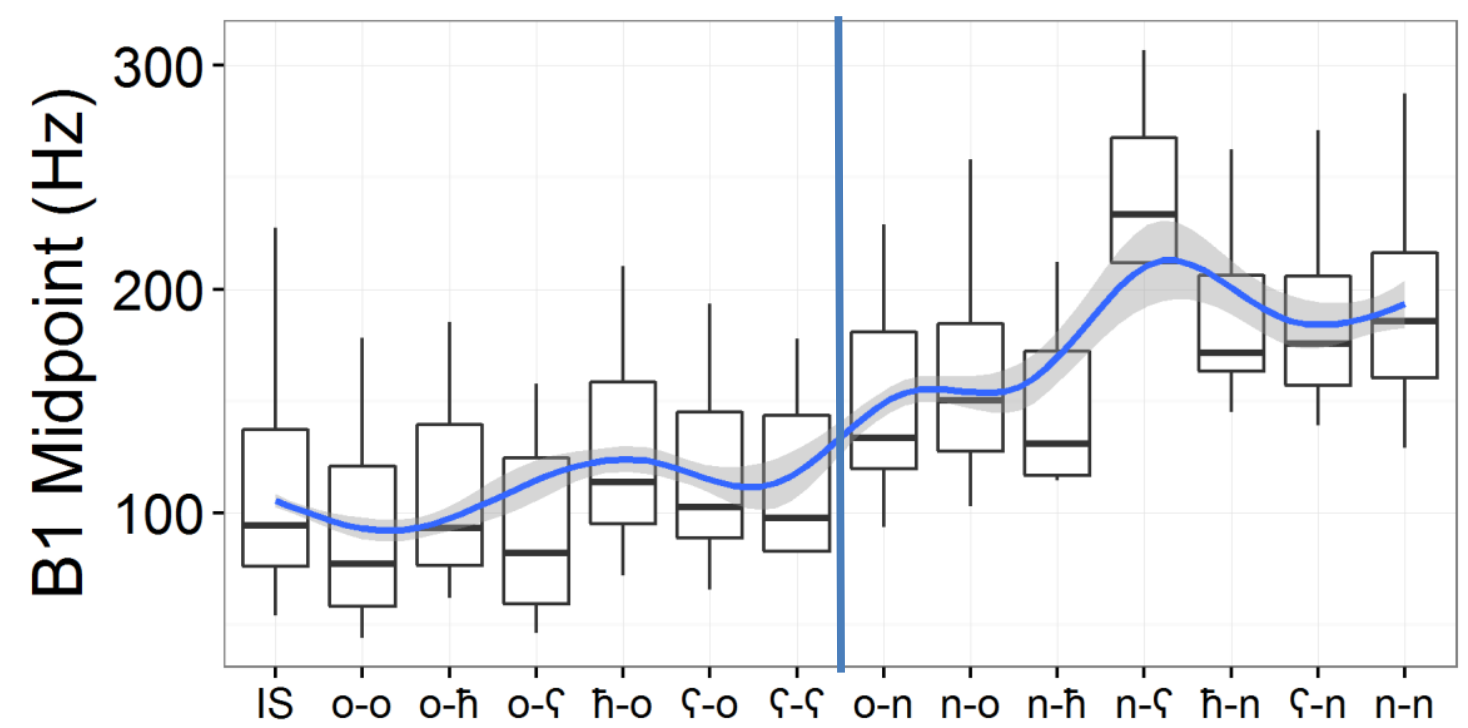

Figure 9: $B 1$ values within oral (left) and nasal (right) contexts at midpoint.

Given that a high $B 1$ can also occur as a result of breathiness in the vowel, as well as any changes linked to laryngeal activity, an acoustic investigation of phonation types was also carried out in this study in order to determine whether our acoustic measures are truly measuring nasalisation.

\subsubsection{Acoustic measure of phonation types: $H 1 *-A 1$}

Here we start with a reminder of how to interpret the relationship between the amplitude of the first harmonic $(H 1)$ and the strongest harmonic of the first formant (A1). According to the literature (e.g. Fry, 1979; Laver, 1980, 1994; Klatt and Klatt, 1990; Trittin and de Santos y Lléo, 1995; Epstein, 2002, Garellek, 2012; Kuang and Keating, 2012), if the first harmonic has the higher amplitude then the resulting voice is believed to be breathy, while when the higher frequency harmonics have higher amplitudes, the resulting voice is considered to be tense/creaky. This would in principle predict a high value for $H 1^{*}-A 1$ if the vowel quality is breathy and a low one if the quality is tense/creaky, but in reality the expected patterns are much more complicated than that as the measure is also influenced by other factors, including nasalisation, which dampens $F 1$ energy and lowers $A 1$ (see Section 3). Figure 10 shows results at onset and offset. 
At onset, and when compared to an intercept-only model, the addition of context showed an improvement to the model fit $\left(\chi^{2}(13)=86.3, p<0.0001\right)$. When comparing the oral context to isolation, there is a significant increase in $H 1^{*}-A 1$, which may be an indication of a more modal to breathy realisation of the vowels in the oral context (Table 7 and Figure 10). Nasals in onset position (n-o and n-n) show a significant increase by 5 to $6 \mathrm{~dB}$ compared to isolation context. This may be an indication that this measure is capturing some level of breathiness that is accompanying nasalisation (Klatt and Klatt, 1990). This may also be due to the decrease in $\mathrm{A} 1$ and concomitant increase in $B 1$ in these contexts. Interestingly, when

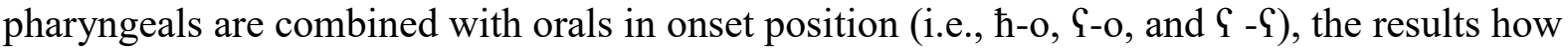
a significant decrease $H 1^{*}$ - $A 1$ by 3 to $5 \mathrm{~dB}$ compared to isolation context. This is indicative of a more tense/creaky realisation of the vowels when preceded by a pharyngeal, and would mirror the descriptions above that the pharyngeal consonants in IA have more of an aryepiglottal manner of articulation that leads to a more constricted larynx (Edmondson et al, 2007; Esling, 2005; Garellek, 2012; Kuang and Keating, 2012). Pharyngeals combined with nasals show an overall decrease in $H 1^{*}-A 1$, with only $\mathrm{Q}$-n showing a significant decrease by around $3 \mathrm{~dB}$. This suggests that even in a nasal context, / $/$ / leads to a more tense/creaky realisation of the vowel.

In offset position, context showed an improvement to model fit when compared to an intercept only model $\left(\chi^{2}(13)=60.8, p<0.0001\right)$. The oral context showed a marginal decrease by $1 \mathrm{~dB}$ compared with isolation, though this was only a tendency $(p=0.09$; see Table 7 and Figure 10). Nasals in offset position (n-n) showed a marginal significant increase by $2 \mathrm{~dB}$ compared to isolation context with o-n showing no differences. Interestingly, when pharyngeals are combined with orals in offset position (i.e., o- $\hbar$, o- $\uparrow, ~ \varsigma-~ \varsigma)$, there is a significant decrease in $H 1^{*}-A 1$ by 7 to $17 \mathrm{~dB}$ compared to the isolation context. The $\mathrm{S}-\mathrm{S}$ context shows once again the lowest value, with a decrease of around $17 \mathrm{~dB}$. This indicates 
that there is a stronger indication of a more tense/creaky realisation of the vowels when followed by pharyngeals. Pharyngeals combined with nasals show a non-significant decrease in $H 1^{*}-A 1$, by 1 to $2 \mathrm{~dB}$, and specifically in $\mathrm{n}-\mathrm{C}$. This again suggests that even in a nasal context, there is a tendency for $/ \mathrm{\&} /$ to lead to a more tense/creaky realisation of the vowel.

Table 7: Results of the Linear Mixed Models for $H 1-A 1^{*}$ at the onset and offset of the vowel in the 13 levels of the fixed effect context. The Intercept is the Isolation context and the $\beta$ coefficients represent the difference between the Intercept and the actual value for the level of the fixed effect. The $p$ values are generated using 7 merTest.

\begin{tabular}{|c|c|c|c|c|c|c|c|c|}
\hline & \multicolumn{4}{|c|}{ Onset } & \multicolumn{4}{|c|}{ Offset } \\
\hline & $\beta$ & Std.Error & $\mathrm{t}$ value & $\operatorname{Pr}(>|t|)$ & $\beta$ & Std.Error & $\mathrm{t}$ value & $\operatorname{Pr}(>|t|)$ \\
\hline (Intercept) & 11.4808 & 1.9648 & 5.843 & 0.000193 & 7.1348 & 1.7096 & 4.173 & 0.001578 \\
\hline O-O & -1.2813 & 0.7601 & -1.686 & 0.09208 & 4.4254 & 0.8283 & 5.343 & $1.05 \mathrm{E}-07$ \\
\hline o- $\hbar$ & -7.4808 & 0.9934 & -7.53 & $8.30 \mathrm{E}-14$ & 3.2077 & 1.0817 & 2.965 & 041 \\
\hline o- & -15.425 & 1.5521 & -9.938 & $<2.00 \mathrm{E}-16$ & 4.3932 & 1.2851 & 3.419 & 0.001557 \\
\hline h-o & -4.1828 & 1.0792 & -3.876 & 0.000114 & -5.5269 & 1.1838 & -4.669 & $3.46 \mathrm{E}-06$ \\
\hline S-O & -2.6415 & & & & & & & $8-05$ \\
\hline S-S & -17.491 & 3.1159 & -5.613 & 2.29 & -3.476 & 1.5825 & -2.197 & 0.027216 \\
\hline o-n & 0.9636 & 0.7681 & & & 4.6545 & 345 & 5.578 & $2.81 \mathrm{E}-08$ \\
\hline n-o & 0.6268 & 1.0161 & 0.617 & & 6.019 & 047 & 5.448 & $5.79 \mathrm{E}-08$ \\
\hline$n-\hbar$ & -0.7174 & 204 & & & 5.5392 & 925 & 15 & 0.020711 \\
\hline$n-\varsigma$ & -2.113 & 1.5159 & -1.393 & & 3.953 & 1.7825 & 2.218 & 0.036375 \\
\hline h-n & 3.9435 & 2.7887 & 1.414 & 0.1 & -0.863 & 3.05 & -0.283 & 0.777265 \\
\hline S-n & 0.5958 & 1.6708 & 0.357 & & -2.7427 & 1.323 & -2.073 & 0.032668 \\
\hline$n-n$ & 2.6838 & 1.3632 & 1.969 & 0.049135 & 5.5957 & 1.4821 & 3.775 & 0.000165 \\
\hline
\end{tabular}




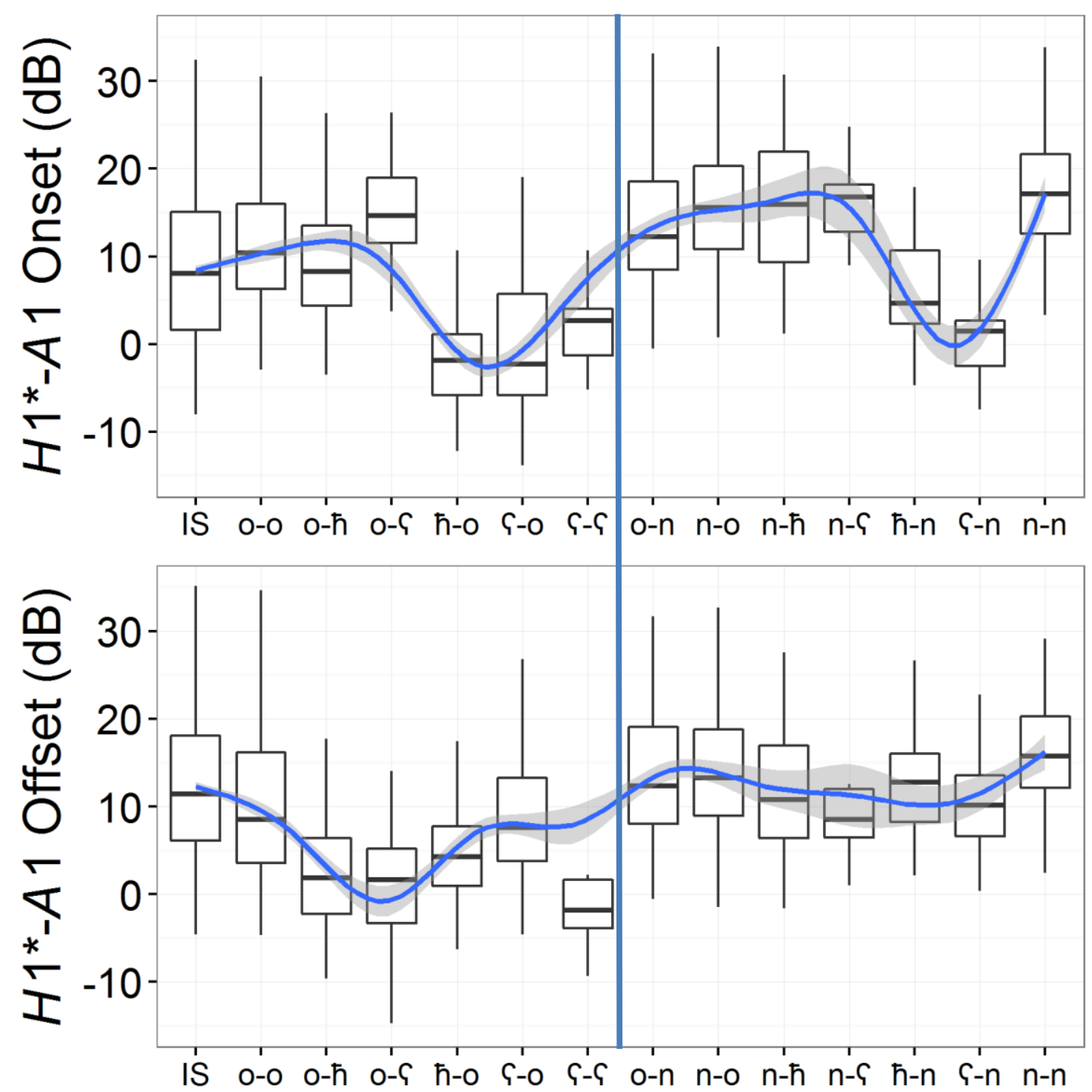

Figure 10: $* H 1-* A 1$ values within oral (left) and nasal (right) contexts at onset (top) and offset (bottom).

\subsection{Principal Components Analyses}

The acoustic results presented above showed that pharyngeals show sporadic weak signs of nasalisation in oral contexts and strong one when associated with nasals, sometimes showing increased nasalisation, as was shown especially robustly for n- . But these results were clearer for some acoustic correlates, e.g., $B 1$, but inconsistent for others, e.g., $A 1-P 0 * / P 1^{*}$. In addition, a voice quality correlate obtained through $H 1^{*}-A 1$ showed pharyngeals to be associated with a tense voice quality that is different from that for true nasals. Our aim in this section is to shed light on the relationships that exist between pharyngeals and nasals by 
presenting the results of an exploratory data reduction technique. We used Principal Components Analyses (PCA) as a dimensionality reduction technique that allows to present the data with a lower number of dimensions than those used. As highlighted above (see Section 6.6), we reoriented the dataset and used each of the 14 contexts as outcomes in this PCA as our aim was to evaluate the degree of closeness/separation of each context. Table 8 (a) presents the results of the first three dimensions that have a variance above 5\% threshold generally used to retain dimensions (Baayen, 2008, p. 121). These three dimensions explained $79.2 \%$ of the variance in the dataset and their combination is representative of the patterns observed in the data.

Figure 11 displays the 3D space represented by the first three dimensions. As shown in Table 8 (b) and Figure 11, all 14 contexts are postively correlated with the first dimension (see correlation results and percentage contributions in Table 8 (b) and loading on the PCA 3D space). This dimension explains $50.8 \%$ of the variance in the data. Table 8 (c) shows the correlation and percentage contribution of each acoustic correlate. B1 Mid is positively correlated with the first dimension while all remaining acoustic correlates are negatively correlated with it. The percentage contribution of all these correlates are relatively high and it is the combination of $A 1-P 1 *$ Offset/Onset, $H 1^{*}-A 1$ Offset followed by $B 1$ Mid that explains most of the patterns on this dimension (percentages around $15 \%$ each). This is a clear indication of the patterning observed in the data whereby the combination of increased $B 1$ Mid and decreased values for nasalisation and voice quality metrics are used by the PCA to cluster this group.

For the second dimension (explaining $20.7 \%$ of the variance), there are two group

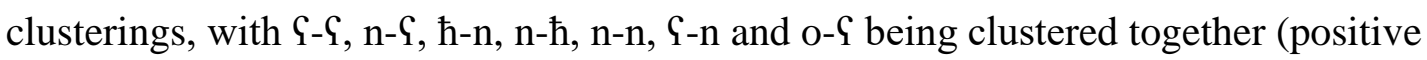
correlation, Table 8 (b), with variable contributions). The two contexts with the highest contributions to this dimension are $\mathrm{n}-\mathrm{\complement}$ and $\mathrm{S}-\mathrm{\complement}$ (followed by the remaining contexts). In terms 
of the acoustic correlates, $B 1$ Mid is negatively correlated with this dimension while all remaining correlates are positively correlated with it (Table 8, (c)). $H 1^{*}$-A1 Onset and $A 1$ $P 0 *$ Onset/Offset positively contribute to the distinction in the first group clustering. Finally, $o-\hbar, o-\varsigma$, - -o, n-o,,$-n$ and n-n are negatively correlated with the third dimension, which explains $7.6 \%$ of the variance in the data (Table 8, parts (c) and (a)). B1 Mid is positively correlated with this dimension while all remaining acoustic correlates are negatively correlated. Interestingly, $H 1^{*}-A 1$ Onset and $A 1-P 0 *$ Onset/Offset have the highest contributions to this dimension, with the reduced values of these correlates being used to cluster the above group. From the 3D PCA space (Figure 11), isolation forms a separate category when compared to the remaining categories and correlates mostly with the third dimension.

When all three dimensions are considered, $९-\subseteq$ is clustered with n- $\complement$, and the same

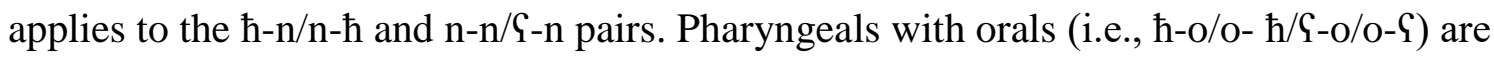
clustered together with o-o and n-o/o-n. Overall, this PCA analysis shows the advantage of examining the combined contribution of all acoustic correlates using the three dimensions on the patterning of the consonantal contexts in this study; it further highlights the subtle increased contribution of pharyngeals to nasalisation in nasal contexts, given that pharyngeal+nasal, but not oral+nasal pattern with nasal+nasal environments.

Table 8: Summary results of the Principal Components Analysis. First part (a) presents the eigenvalues for the first three dimensions with the 14 contexts as outcomes. The second part (b) shows the correlation between each context and dimension and (percentage contribution). The third part (c) shows the estimates for each acoustic correlate and its correlation with each dimension and (percentage contribution).

\begin{tabular}{|c|c|c|c|}
\hline (a) & Dimension 1 & Dimension 2 & Dimension 3 \\
\hline Variance & 7.1 & 2.9 & 1.1 \\
\hline $\begin{array}{c}\text { Variance (\%) } \\
\text { Cummulative Variance } \\
(\%)\end{array}$ & 50.8 & 20.7 & 7.6 \\
\hline (b) & 50.8 & 71.5 & 79.2 \\
\hline Isolation & $0.36(1.86)$ & $-0.32(3.65)$ & $0.71(47.49)$ \\
\hline o-o & $0.69(6.73)$ & $-0.51(8.97)$ & $0.27(6.86)$ \\
\hline
\end{tabular}




\begin{tabular}{|c|c|c|c|}
\hline $0-\hbar$ & $0.85(10.09)$ & $-0.33(3.76)$ & $-0.15(2.08)$ \\
\hline $0-\Upsilon$ & $0.68(6.56)$ & $0.35(4.28)$ & $-0.21(4.16)$ \\
\hline ћ-o & $0.83(9.77)$ & $-0.4(5.6)$ & $0.05(0.27)$ \\
\hline S-o & $0.86(10.29)$ & $-0.31(3.39)$ & $-0.1(0.89)$ \\
\hline ६-ৎ & $0.5(3.46)$ & $0.67(15.47)$ & $0.32(9.72)$ \\
\hline o-n & $0.76(8.07)$ & $-0.46(7.16)$ & $0.16(2.49)$ \\
\hline n-o & $0.78(8.66)$ & $-0.32(3.48)$ & $-0.24(5.44)$ \\
\hline$n-\hbar$ & $0.61(5.24)$ & $0.59(12.2)$ & $0.01(0.01)$ \\
\hline n- $-\uparrow$ & $0.54(4.04)$ & $0.7(16.93)$ & $0.3(8.42)$ \\
\hline ћ-n & $0.65(5.84)$ & $0.62(13.37)$ & $0.03(0.09)$ \\
\hline §-n & $0.83(9.77)$ & $0.21(1.55)$ & $-0.23(4.88)$ \\
\hline n-n & $0.83(9.62)$ & $0.07(0.18)$ & $-0.28(7.2)$ \\
\hline \multicolumn{4}{|l|}{ (c) } \\
\hline B1Mid & $2.59(14.56)$ & $-1.09(14.7)$ & $1.35(14.73)$ \\
\hline$A 1-P 0^{*}$ Onset & $-0.41(13.55)$ & $0.18(15.13)$ & $-0.23(16.44)$ \\
\hline$A 1-P 0^{*}$ Offset & $-0.38(13.13)$ & $0.17(14.87)$ & $-0.22(16.26)$ \\
\hline$A 1-P 1^{*}$ Onset & $-0.36(15.41)$ & $0.14(14.11)$ & $-0.16(12.17)$ \\
\hline$A 1-P 1 *$ Offset & $-0.38(15.43)$ & $0.15(13.08)$ & $-0.17(11.83)$ \\
\hline$H 1{ }^{*}$-A1 Onset & $-0.54(12.65)$ & $0.25(15.7)$ & $-0.33(17.21)$ \\
\hline$H 1{ }^{*}$-A1 Offset & $-0.52(15.26)$ & $0.2(12.42)$ & $-0.23(11.36)$ \\
\hline
\end{tabular}




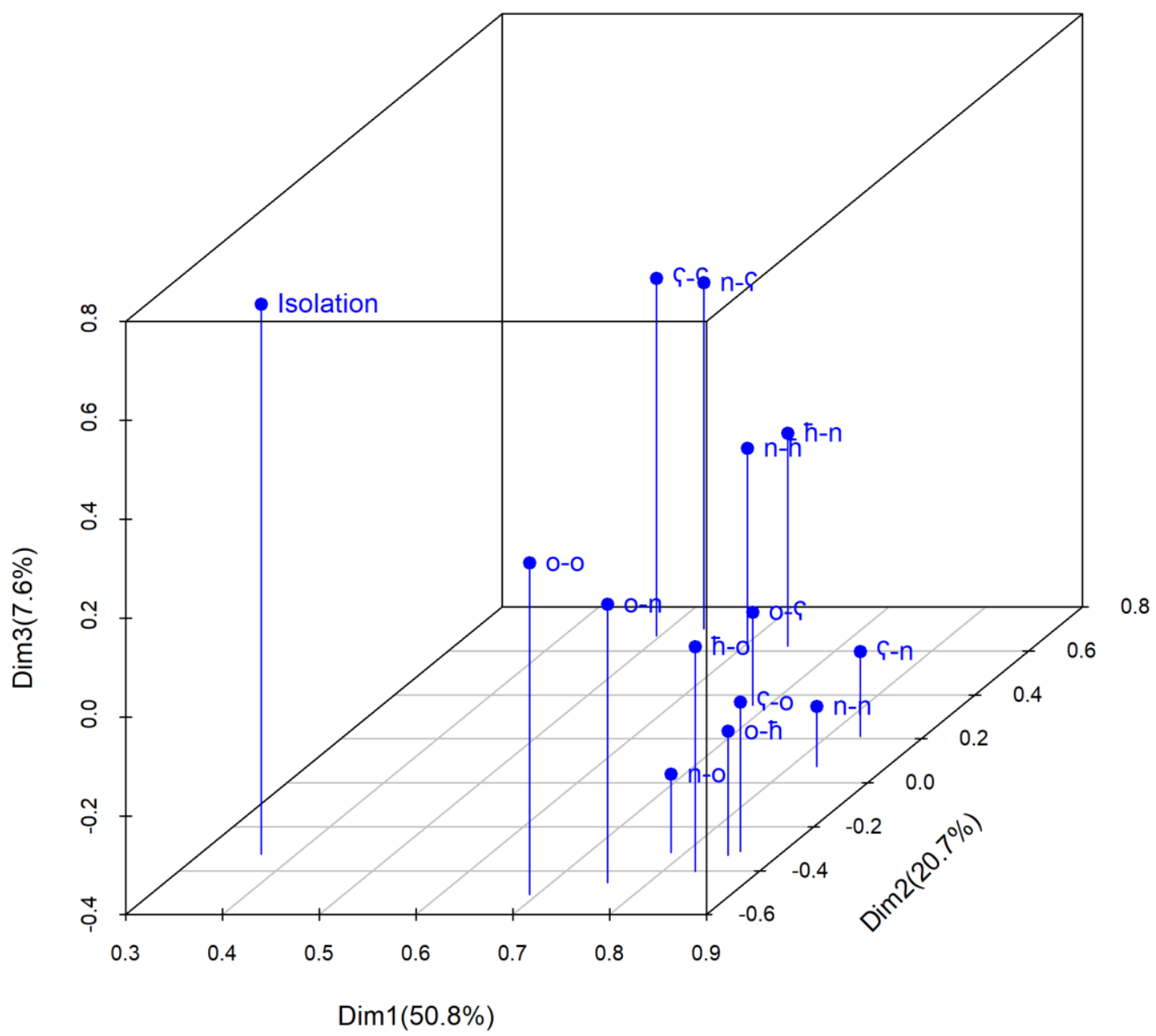

Figure 11: 3-Dimension Principal Components Analysis (PCA) space with the first three dimensions with (percent variance explained). Each of the 14 levels of context are represented on this 3-D space. The vertical lines show the correlations of each context with the three dimensions.

\section{Summary of results}

This study examined a number of impressionistic and acoustic measures which are meant to capture the influence of perceived nasalisation in vowels, with the aim of finding out whether pharyngeal consonants in Arabic are produced with nasalisation, and whether nasalisation has spread to oral contexts as well. Data from nasal, oral and pharyngeal contexts were collected from IA speakers, including pharyngeal+oral and pharyngeal+nasal contexts. The auditory analysis of the data revealed a main separation between oral contexts (including oral, 
isolation, and pharyngeal+oral) on the one hand and nasal contexts (including pharyngeal+nasal and nasal) on the other. A small proportion of the data within the mainly oral group still, however, received nasal ratings, especially the voiced pharyngeal tokens, but also vowels in isolation. This is not surprising when considering that nasalisation can occur in oral contexts and may be due to passive velum lowering.

When combined with a nasal consonant, pharyngeal consonants exhibited a nasalisation effect on the surrounding vowel. Nasalisation ratings were higher for nasals combined with voiced pharyngeals than nasals combined with other contexts, suggesting an increased nasalisation effect in the presence of voiced pharyngeals. This percept was also manifested in the acoustic analysis and suggests that nasalisation may indeed be a feature of at least voiced pharyngeal production. Auditory impressions of voice quality yielded very few instances of perceived breathiness, with nasal and oral contexts being perceived as mainly modal while pharyngeal+oral and pharyngeal+nasal contexts were mainly perceived as tense.

Four acoustic measures were chosen as the focus for this study; the first three, $A 1-P 0^{*}$, $A 1-P 1^{*}$ and $B 1$ were used to investigate the potential influence of nasal coupling and/or high laryngeal airflow in the production of pharyngeals on the amplitude of the first formant and its bandwidth, as well as on any resulting extra peaks in the spectrum. Given that the presence of breathiness can influence $A 1-P 0 * / A 1-P 1^{*}$ and $B 1$ patterns in the same way as nasalisation, a third measure, $H 1^{*}-A 1$, was chosen to test for the presence of breathiness in pharyngeal contexts. A summary of results from the three measures is provided in this section, followed by a wider discussion in Section 7.

A general view of the $A 1-P 0^{*}$ results shows this measure to be reliable for distinguishing oral and nasal contexts, and corroborates the auditory analysis which placed pharyngeal+oral contexts with the remaining oral contexts, while pharyngeals combined with nasals showed various degrees of nasalisation; the acoustic manifestation of this effect were, 
predictably, more variable than its auditory manifestation, but this was compounded by the finding that $A 1-P 0^{*}$ for pharyngeal+oral were generally very high, significantly higher than oral-oral contexts. This required a larger drop in amplitude difference in the nasal contexts for the values to be comparable to the other nasal contexts, something that was only achieved for $/ \mathrm{S} /$ in the $\mathrm{n}-\mathrm{S}$ context. Measurements of $A 1-P 0^{*}$ at offset showed a reduced nasal/oral distinction, but within it the pharyngeal+oral and pharyngeal+nasal patterns remained the same. Here again, the extremely high amplitude values for the pharyngeals in oral contexts, especially / $/$ /, required a significant drop in the nasal contexts for it to match the values found for the other nasal contexts. The $A 1-P 1^{*}$ measure at onset was also reliable for distinguishing oral and nasal contexts, but the values for nasals were higher than might be expected for this measure. The same is true at offset, with only the nasal-nasal context having mean $A 1-P 1^{*}$ values that were lower than $10 \mathrm{~dB}$. The pharyngeal+oral contexts patterned more with oral contexts, but o- $\hbar$ interestingly patterned with nasals. There was more variability and a less clear pattern for pharyngeal+nasal contexts due to the reduced number of vowel contexts in the high vowel environment and no data for / / / combined with a nasal.

Results for $B 1$ were more robust and showed an overall distinction between nasal and non-nasal contexts, whereby the former had higher $B 1$ values. Here, pharyngeal-oral contexts were significantly different from other oral contexts, for both voiced and voiceless pharyngeals. They displayed higher $B 1$ values that could be interpreted as displaying subtle nasalisation effects, but were still significantly lower than for nasal contexts. As was found for the $A 1-P 0^{*}$ and $A 1-P 1^{*}$ measures, and although non-statistically significant, a combination of a nasal and a pharyngeal context triggered increased nasalisation compared with nasal+oral contexts; this was especially true for /n-S/, which had the highest mean value of all contexts, but $€-n$ and $\hbar-n$ had values comparable to $n-n$ as well. 
Results of the $H 1^{*}-A 1$ measure showed a marked departure from the way pharyngeal contexts patterned for the other measures. Here, pharyngeal consonants exhibited the lowest values both in pharyngeal+oral and pharyngeal+nasal contexts, suggesting high levels of laryngealisation (tense phonation) in these contexts. Oral and nasal contexts had significantly higher $H 1^{*}-A 1$ than pharyngeals, with nasals exhibiting the highest values. The differences between the four main contexts (oral/isolation, pharyngeal+oral, pharyngeal+nasal and nasal) were more pronounced at onset than offset, but the patterns were generally the same. Finally, pharyngeal / $\mathrm{S} /$ tended to have lower $H 1^{*}-A 1$ values than $/ \mathrm{h} /$, indicating more laryngealisation.

A PCA analysis combining all acoustic measures was used as a data reduction technique and showed that the first three dimensions explained $79.2 \%$ of the variance in the data. Within these dimensions, pharyngeal+oral contexts cluster with other oral and oral+nasal contexts (though in a sub-group of their own), but pharyngeal+nasal contexts cluster with nasal contexts, with each of $\hbar-n / n-\hbar, \varsigma-\varsigma / n-\varsigma$, and $n-n / \varsigma-n$, forming a sub-group within that cluster. In a nasal context, the voiced pharyngeal appears to consistently adopt a nasal configuration and to contribute to increased nasalisation. Taken together, these results only partially corroborate findings from the literature which suggest that pharyngeals in IA are accompanied by nasalisation (e.g. Butcher and Ahmad, 1987; Ghazeli, 1977; Laradi, 1983).

\section{Discussion}

This study has looked at pharyngeal consonants from a different angle to what is normally focussed on in the Arabic literature; building on research which suggests that pharyngeal consonants in IA have an epiglottal place of articulation with laryngeal constriction and raising alongside tongue retraction and mid to low pharyngeal constriction (Butcher and Ahmad, 1987, Edmondson et al, 2007; Esling, 2005; MacCurtain, 1981), the study examined the auditory and acoustic effect of such articulation with particular reference to nasalisation. 
Small-scale articulatory studies in the past had found pharyngeals to be accompanied with velum lowering and occasional nasal airflow or a percept of nasalisation (Butcher and Ahmad, 1987; Ghazeli, 1977; Laradi, 1983), but the results were inconsistent and varied across speakers. The current study found emerging evidence of spectral effects that seem to be responsible for a perceptual effect of nasality in vowels surrounding pharyngeal consonants, but these were only consistent in the presence of nasal consonants, and the nasalisation effect seems to be boosted when a voiced pharyngeal is present. Below we evaluate the effectiveness of the chosen spectral measures for capturing nasalisation in pharyngeal contexts and reflect on what the differential results for $/ \mathrm{S} /$ and $/ \mathrm{h} /$ may mean for which aspect of the articulation of these sounds may be triggering audible nasalisation and/or actual velum lowering.

\subsection{Evaluating spectral measures of nasalisation in the context of pharyngeals}

In terms of the individual spectral measures that we examined, $B 1$ was the most effective in showing slight nasalisation in pharyngeals, even in oral contexts. In fact, this measure divided the contexts into four categories which show gradation in the degree of nasalisation according to the following pattern: lowest in the oral and oral-pharyngeal contexts, slight in the pharyngeal-oral contexts, higher in the oral-nasal and nasal-oral contexts (but including $\mathrm{n}-\hbar$ ),

and highest in nasal-nasal, pharyngeal-nasal and nasal-ৎ. The latter context consistently showed the highest degree of nasalisation, including in results of other acoustic measures.

While the $A 1-P 0^{*}$ and $A 1-P 1^{*}$ proved generally sensitive to the oral-nasal separation, the former was generally more robust for distinguishing oral and nasal environments. Given that $A 1-P 0^{*}$ was used for low vowels and $A 1-P 1^{*}$ for high vowels, this might explain why low vowels are more susceptible to nasalisation than high vowels (Sampson, 1999). Importantly, though $A 1-P 0^{*}$ and $A 1-P 1^{*}$ showed more inconsistent results for the pharyngeal contexts, whether in oral or in nasal contexts. One reason for this is that these measures were 
also indexing pharyngealisation, and the amplitude correlates for that go counter to those of nasalisation. While nasal environments tend to lead to a reduction in overall vowel intensity, pharyngeal articulations typically lead to a rise in the frequency and amplitude of $F 1$ (Al-Ani, 1970; Al-Siraih, 2013; Butcher and Ahmad). In the case of laryngealised pharyngeals, this also tends to be accompanied by a drop in frequency and amplitude of $f 0$ (Heselwood, 2007), leading to a larger difference between $A 1$ and $P 0$. In this study the effect was most prominent in voiced pharyngeal+oral environments, where both $A 1-P 0^{*}$ and $A 1-P 1^{*}$ measures for $\mathrm{Y}-\mathrm{o}$ at onset, o- $\complement$ at offset and $\varsigma-\varsigma$ were $20 \mathrm{~dB}$ or higher, reaching nearly $30 \mathrm{~dB}$ for o- $\subseteq$ at $A 1-P 1 *$ offset. The effect was occasionally seen for $/ \hbar /$ too (e.g. $A 1-P 0 *$ offset for o- $\hbar$ ), but never as prominent as for $/ \mathcal{S} /$. This leads us to reflect on the differences in the degree of nasalisation that we witnessed for the voiced and voiceless pharyngeals, which we return to below. Importantly though, the pharyngealisation effect needs to be taken into account when scrutinising the $A 1-P 0 *$ and $A 1-P 1 *$ patterns for nasal+pharyngeals contexts. These were still comparable to the amplitude values for the other nasal environments, suggesting that extreme A1 amplitude lowering took place compared with pharyngeal+oral environments along with possible boosting of the amplitude of the extra peaks. Therefore, while $A 1-P 0^{*}$ and $A 1-P 1^{*}$ patterns for pharyngeal+nasal environments were not significantly different from those of other nasal environments, this does not rule out added contribution of nasalisation in pharyngeal+nasal environment, especially for the voiced pharyngeal.

The $H 1^{*}-A 1$ measure was crucial for establishing whether any auditory percept of nasalisation in pharyngeal contacts was due to laryngeal friction producing a percept of breathy voice (Beddor, 1993; Garellek, Ritchart and Kuang, 2016; Matisoff, 1975). Our impressionistic results did not yield breathiness ratings in pharyngeal contexts, and the tense articulation of IA pharyngeals is incompatible with a breathy phonation. In fact, the epilaryngeal constriction in pharyngeals, together with larynx raising, typically leads to a 
tight constriction in the glottis and results in a tense or pressed voice quality (Al-Tamimi, J., 2017; Esling, 2005; Heselwood, 1997; Klatt and Klatt, 1990; Moisik 2013). This was corroborated by our $H 1^{*}-A 1$ results, showing a sharp decrease in this spectral tilt measure in pharyngeal environments, especially in onsets. It therefore seems that IA pharyngeals show only certain characteristics of breathiness and/or nasalisation such the introduction of extra poles in the spectrum, a wider first band frequency, and a reduced amplitude of $F 1$, but not others such an increased amplitude of the first harmonic relative to higher frequency harmonics or aspiration noise at high frequencies (Klatt and Klatt, 1990; Sampson, 1999).

Nasality and breathiness do not have to co-occur, and in fact Garellek et al (2016) show data from three languages where nasality (in this case presence or absence of velum lowering) and phonation (breathiness or tenseness) are orthogonal and vary from one language to another. While nasal and breathy vowels share common features like increased first formant bandwidth, overall low-frequency energy, and a stronger first harmonic, speakers can enhance nasals with breathy voice or enhance breathiness with what Ohala and Ohala (1993) refer to as spontaneous nasalisation, but the two can also occur independently from each other. This is common in languages where laryngeals do not contrast in terms of nasalisation, but perhaps as a result of that tends to apply in unpredictable and varied ways.

While the current study does not use articulatory techniques, the inclusion of a relatively larger sample of speakers than is normally possible in an articulatory study has allowed us to report on the acoustic manifestations which seem to be responsible for perceptual nasality regardless of the articulatory strategies used by the speakers. It is quite possible that our speakers are using a variety of strategies for pharyngeal production, and that some are at a more advanced stage of velum lowering than others. While space limitations have not allowed us to discuss individual differences here, some speakers were indeed found to produce lower $A 1-P 0 * / A 1-P 1^{*}$ and higher $B 1$ values, and potential dialectal differences 
emerged as well (Alsiraih, 2013). More evidence for nasalisation in pharyngeal contexts may have been found if we had varied the prosodic context in which the target words were elicited. In this study, all target words were in a nuclear stressed position, which typically leads to articulatory strengthening and an increase in the laryngeal and pharyngeal constriction, potentially leading to a stronger velar closure as well (Fougeron and Keating, 1997).

\subsection{Comparing nasalisation results for the voiced and the voiceless pharyngeals: a} window into their varying articulation?

Descriptions of Iraqi Arabic pharyngeals show more consistency in the realisation of $\hbar$, which is described as a pharyngeal fricative, than in the realisation of $\subseteq$, which tends to be realised with a lower epilaryngeal constriction and to vary in manner from an approximant to a fricative and even a stop (e.g. Al-Ani, 1970; Butcher and Ahmad, 1987; McCarthy and Raffouli, 1964; MacCurtain, 1981; however, cf. Edmondson et al, 2007, who finds laryngeal constriction of the aryepiglottic folds in $/ \hbar /$ too). The less variable nature of $/ \hbar /$ was corroborated in a more recent acoustic study by the third author (Alsiraih, 2013), who found acoustic and impressionistic evidence for frication in $/ \mathrm{h} /$, whereas $/ \mathrm{S} /$ tended to vary between an approximant, a fricative, and a stop. At the same time though, early mentions of nasalisation in pharyngeals in IA refer to / $\mathrm{h} /$ (Butcher and Ahmad, 1987) as well as / $\mathrm{G} /$ (Mahdi, 1985), suggesting that different sources of nasalisation may be at play when they are encountered for each sound. For the voiceless pharyngeal, the more fricative-like manner suggests that, where auditory nasalisation is present, this is more likely to be due to the type of perceptual nasalisation that is triggered by high laryngeal airflow, with added energy loss at the glottis due to a larger glottal opening (Hanson, 1996; Hanson and Chuang, 1999). This may lead to a rise in the $F 1$ frequency of neighbouring vowels while lowering their amplitude and increasing their bandwidth, especially for $F 1$ (Ohala and Ohala, 1993; Ohala, 1975). 
Coupled with pole-zeros in the higher frequencies of the spectrum, a perceptual effect of nasality is created. This would also explain why nasalisation in $/ \hbar /$ most apparent in the $B 1$ measure, which is sensitive to laryngeal effects.

For the voiced pharyngeal, there are more possible sources of nasalisation: first, if articulation for $/ \mathrm{S} /$ is indeed lower than for $/ \mathrm{h} /$, this might increase the possibility of actual velum lowering as a result of the action of the root of the tongue and/or the epilaryngeal constriction (Dixit et al, 1987; Delattre, 1971; Laver, 1980; Sampson, 1999). The possibility of actual velum lowering in the voiced pharyngeal can explain why nasal+voiced pharyngeal contexts show more perceived nasalisation than other nasal contexts; if both the nasal and the pharyngeal are produced with a lowered velum, this increases the effect of nasalisation on neighbouring vowels. This, however, remains a debatable issue requiring a future articulatory study, since work by Laradi (1983) on Libyan Arabic shows more velum contact with the naso-pharynx for $/ \mathrm{S} /$ than $/ \hbar /$. Second, if, as commonly suggested in the literature, $/ \mathrm{S} /$ involves more than one constriction, then perceptual nasalisation may result from the creation of a laryngeal sub-cavity. Such cavity can be created in at least two ways: (a) through actions of the epiglottis: here, the tip of the epiglottis forms a complete closure with the back of the pharyngeal wall (Laufer and Condax, 1979; 1981; MacCurtain; 1981) and the base of the epiglottis forms a constriction with the aryepiglottic folds (Esling 2005; Heselwood, 1997; Laver, 1980; Zeroual et al, 2008). The varying degree of constriction also explains the inconsistent patterns found in this study; if there is only narrow constriction between the tip of the epiglottis and the posterior pharyngeal wall, no tight cavity is formed; (b) through actions of the tongue and larynx: here, the root of the tongue may also retract towards the mid-pharyngeal, the larynx may raise for efficient laryngeal sphinctering (Esling, 2005; MacCurtain; 1981), and the glottis may form a complete closure for a glottal stop (Al-Ani, 1970). Once again, tight articulations are required for a separate cavity to form in the 
pharyngeal area between the tongue root and the glottis, leading to potential variability in nasalisation.

The above possibilities for / / / make for a very complex set of articulations and we still do not fully understand how each of them may vary across various linguistic contexts, speakers and dialects, and whether they co-occur (e.g. Laradi’s 1983 work on Libyan Arabic; Laver's 1980 on pharyngealised voice, etc.). The variability in articulation, however, also seems to offer more opportunities for nasalisation in the case of the voiced pharyngeal, and helps explain the consistent pattern we found for $/ \mathcal{S} /$ to show more nasalisation. If variable nasalisation in pharyngeals were to be picked up by listeners and phonologised, this study would predict that the effect would be seen first in voiced pharyngeals before spreading to voiceless ones. In other words, if listeners start associating the perceived nasalisation on the vowel with a neighbouring nasalised pharyngeal/laryngeal, then they might interpret that as target nasality and start adopting a lowered velum in their production (Matisoff, 1975; Ohala, 1975; Sampson, 1999).

\subsection{Direction of nasalisation and pharyngeal spread}

The result of the progressive effect of nasals is an interesting one and places IA in the group of languages where the domain of nasalisation is primarily onset-vowel rather vowel-coda (e.g. Botma, 2004 for evidence from Sundanese; Hyman, 1972 for evidence from Kwa). A regressive effect of nasalisation has been commonly reported in languages (e.g. Blust, 1997; Ferguson, 1975), including cases where a weak coda nasal typically leads to a transfer of the nasal feature to the preceding vowel, which may over time become nasalised, replacing the final nasal (e.g. Beddor, 2009; Botma, 2004; Sampson, 1999; Ohala, 1981). However, a similar outcome can be seen in progressive nasalisation; for instance, earlier studies of Semitic languages like Gurage (Hetzron, 1969) show that, if gutturals turn into laryngeals, they could indeed trigger progressive nasalisation in vowels around them (and in some cases 
turn into nasals if progressive nasalisation is blocked). Pharyngeal and pharyngealised (emphatic) sounds in Arabic generally exhibit a predominantly rightward spread of pharyngealisation (Ghazeli, 1977), which is compatible with the progressive nasalisation that was found in this study. However, the domain of emphasis (neighbouring sound, syllable, word, and morpheme), direction and type of segment that blocks the spread are subject to dialectal differences (e.g. Younes, 1993). This highlights the importance of careful description of language differences and dialectally grounded variation in helping us understand the range of phonetic patterns that may become phonologised (Botma, 2004; Clumeck, 1975).

\section{Conclusion}

This paper makes three main contributions to the study of pharyngeals and nasalisation: first, it provides a systematic and robust analysis of the acoustic and impressionistic patterns in the production of IA pharyngeals in various oral and nasal contexts. In doing so, the study paves the way for future articulatory work and facilitates studies that seek to model articulatoryacoustic relations in the production of pharyngeals. Second, the analyses provide a current account of nasalisation and breathiness in the different segmental contexts that were investigated, and successfully tease apart the nasalisation patterns found in pharyngeals from any phonation characteristics which might accompany their articulation. Third, the study offers a great potential for future studies documenting further development of what could be seen here as the start of nasalised pharyngeals in IA. The ambiguous acoustic patterns that were found in the realisation of pharyngeals make room for reinterpretation of intended articulations by listeners and the development of new sound representations across generations, leading to sound change (Ohala, 1975; Botma, 2004). This would provide an understanding of how certain dialectal differences emerge and develop over time, as well as the history of the development of some of the more subtle types of differences in the 
realisation of certain sound categories across languages and dialects. As more articulatory work is becoming available, the involvement of the larynx in pharyngeal articulation is increasingly being reported for other dialects too (Esling, 2005; Heselwood and Al-Tamimi, F., 2011; and see Al-Tamimi, J., 2017 for acoustic work), and it would be interesting to see more studies looking at epilaryngeal effects of pharyngeal production on perceptual nasalisation and nasal coupling, both active and passive.

\section{References}

Agresti, A. (2002) Categorical data analysis (second edition). New York: John Wiley \& Sons.

Al-Ani, S.H. (1970) Arabic Phonology: An Acoustical and Physiological Investigation. Berlin \& New York: Mouton De Gruyter.

Alsiraih, W. (2013) Voice Quality Features in the Production of Iraqi Arabic Speakers. PhD Thesis, Newcastle University.

Al-Tamimi, J. (In Press) 'Revisiting Acoustic Correlates of Pharyngealization in Jordanian and Moroccan Arabic: Implications for Formal Representations'. Laboratory Phonology: Journal of the Association for Laboratory Phonology, 8(1): 1-40.

Al-Tamimi, J. and Khattab, G. (2011) 'Multiple cues for the singleton-geminate contrast in Lebanese Arabic: acoustic investigation of stops and fricatives', $17^{\text {th }}$ International Congress of Phonetic Sciences (ICPhS), Hong Kong (17-21 August), pp. 212-215.

Al-Tamimi, J. and Khattab, G. (2015) 'Acoustic cue weighting in the singleton vs geminate contrast in Lebanese Arabic: the case of fricative consonants', Journal of the Acoustical Society of America, 138 (1), pp. 344-360. doi:10.1121/1.4922514.

Amino, N. and Osanai, T. (2012) 'Speaker characteristics that appear in vowel nasalisation and their change over time', The Acoustical Society of Japan, 33(2), pp. 96-105. 
Bartko, J.J. (1966). The intraclass correlation coefficient as a measure of reliability. Psychological Reports, 19, 3-11.

Bates, D., Maechler, M., Bolker, B. and Walker, S. (2015) 'Fitting Linear Mixed-Effects Models Using lme4'. Journal of Statistical Software, 67(1), pp. 1-48.

Beck, J. (2005) 'Perceptual analysis of voice quality: the place of Vocal Profile Analysis', In W.J. Hardcastle and J. Mackenzie (eds.) A Figure of Speech: a Festchrift for John Laver. Laurence Erlbaum, London, pp. 285-322.

Beddor, P. S. (1983) Phonological and Phonetic Effects of Nasalization on Vowel Height. Indiana: Indiana University Press.

Beddor, P. S. (2007) 'Nasal consonants and nasalisation: the relation between segmental and coarticulatory timing', $16^{\text {th }}$ International Congress of Phonetic Sciences (ICPhS), Saarbrucken (6-10 August), pp. 249-254.

Beddor, P. S., \& Krakow, R. A. (1999). 'Perception of coarticulatory nasalization by speakers of English and Thai: Evidence for partial compensation'. The Journal of the Acoustical Society of America, 106(5), pp. 2868-2887.

Bell-Berti, F. (1993) 'Understanding velic motor control: studies of segmental context'. In M. K. Huffman and R. A. Krakow (eds). Nasals, Nasalization, and the Velum. pp. 63-85.

Bellem, A. (2007) Towards a Comparative Typology of Emphatics: Across Semitic and into Arabic Dialect Phonology. PhD Thesis. University of London: School of Oriental and Arabic Studies.

Berger, M. A. (2007) Measurement of Vowel Nasalization by Multi-Dimensional Acoustic Analysis. M.A. Thesis, University of Rochester.

Blust, R. (1997) 'Nasals and nasalization in Borneo'. Oceanic Linguistics, 36 (1), pp. 149179. 
Botma, E.D. (2004) Phonological aspects of nasality: An element-based dependency approach. $\mathrm{PhD}$ Thesis, University of Amsterdam.

Boersma, P. and Weenink, D. (2017) Praat: doing phonetics by computer (Version 6.0.23) [Computer program]. Available at: http://www.praat.org/ (Accessed: 24 January 2017).

Butcher, A. and Ahmad, K. (1987) 'Some acoustic and aerodynamic characteristics of pharyngeal consonants in Iraqi Arabic', Phonetica, 44, (3), pp. 156-172.

Catford, J. C. (1977) Fundamental Problems in Phonetics. Edinburgh: Edinburgh University Press.

Cicchetti, D. V. (1994). Guidelines, criteria, and rules of thumb for evaluating normed and standardized assessment instruments in psychology. Psychological Assessment, 6(4), 284-290.

Chen, M. Y. (1995) 'Acoustic parameters of nasalised vowels in hearing-impaired and normal-hearing speakers', Journal of the Acoustical Society of America, 98, (5), pp. 2443-2453.

Chen, M. Y. (1997) 'Acoustic correlates of English and French nasalised vowels', Journal of the Acoustical Society of America, 102, (4), pp. 2360-2370.

Chen, M. Y. (2000) 'Acoustic analysis of simple vowels preceding a nasal in Standard Chinese', Journal of Phonetics, 28, pp. 43-67.

Chen, M. Y., Stevens, K. N., Kuo, H.-K. J. and Chen, H. (2000) 'Contributions of the study of disordered speech to speech production models', Journal of Phonetics, 28, pp. 303-312.

Chen, N. F., Slifka, J. L. and Stevens, K. N. (2007) 'Vowel nasalisation in American English: acoustic variability due to phonetic context', $16^{\text {th }}$ International Congress of Phonetic Sciences (ICPhS), Saarbrucken (6-10 August), pp. 905-908. 
Clumeck, H. (1975) 'A cross-linguistic investigation of vowel nasalisation: an instrumental study' in Ferguson C. A., L. M. Hyman and J. J. Ohala (eds.), Nasalfest: Papers from a Symposium on Nasals and Nasalization. USA: Stanford University, pp. 133-152.

Cohen, J. (1988) Statistical Power Analysis for the Behavioural Sciences. 2nd edition. Routledge Academic.

Cohn A.C. (1993) 'Nasalisation in English: phonology or phonetics'. Phonology, 10 (1), pp. 43-81.

Christensen (2015). Christensen, R. H. B. (2015) ordinal - Regression Models for Ordinal Data. R package version 2015.6-28. http://www.cran.r-project.org/package=ordinal/.

Delattre, P. (1971) 'Pharyngeal features in the consonants of Arabic, German, Spanish, French and American English'. Phonetica, 23, pp. 129-155.

Dixit, R. P., Bell-Berti, F., \& Harris, K. S. (1987) 'Palatoglossus activity during nasal/nonnasal vowels of Hindi'. Phonetica, 44(4), 210-226.

Edmondson, J. A., Esling, J. H., Harris, J. G. and Huang, T. (2005) 'A laryngoscopic study of glottal and epiglottal/pharyngeal stop and continuant articulations in amis - an Austronesian language of Taiwan', Language and Linguistics, 6 (3), pp. 381-396.

Edmondson, J. A., Padayodi, C. M., Hassan, Z. M. and Esling, J. H. (2007) 'The laryngeal articulator: source and resonator', $16^{\text {th }}$ International Congress of Phonetic Sciences, Saarbrucken (6-10 August), pp. 2065-2068.

Epstein, M. A. (2002) Voice Quality and Prosody in English. PhD Thesis, University of California.

Esling, J. H. (1999) 'The IPA Categories "Pharyngeal" and "Epiglottal": laryngoscopic observations of pharyngeal articulations and larynx height', Language and Speech, 42 (4), pp. 349-372. 
Esling, J. H. (2005) 'There are no back vowels: the Laryngeal Articulator Model', Canadian Journal of Linguistics, 50, (1/2/3/4), pp. 13-44.

Fry, D. B. (1979) The Physics of Speech. Cambridge: Cambridge University Press.

Fujimura, O. and Lindqvist, J. (1971) 'Sweep-tone measurements of vocal-tract characteristics', Journal of the Acoustical Society of America, 49, (2 (Part 2)), pp. 541558.

Gamer, M., Lemon, J. and Singh, I.F.P (2012) irr: Various Coefficients of Interrater Reliability and Agreement. $\mathrm{R}$ package version 0.84 . https://CRAN.Rproject.org/package=irr.

Garellek, M. (2012) 'The timing and sequencing of coarticulated non-modal phonation in English and White Hmong'. Journal of Phonetics, 40(1), 152-161. doi: doi:10.1016/j.wocn.2011.10.003.

Ghazeli, S. (1977) Back Consonants and Backing Coarticulation in Arabic. PhD thesis. University of Texas.

Glass, J. R. and V. W. Zue (1985) 'Detection of nasalised vowels in American English'. Institute of Electrical and Electronics Engineers (IEEE): 1569-1572.

Hallgren, K. A. (2012) Computing inter-rater reliability for observational data: an overview and tutorial. Tutorials in quantitative methods for psychology, 8(1), 23-34.

Hanson, H. M. (1996) 'Glottal characteristics of female speakers: acoustic correlates.' Journal of the Acoustical Society of America, 101(1), pp. 466-481.

Hanson, H. M. and E. S. Chuang (1999) 'Glottal Characteristics of Male Speakers: Acoustic correlates and comparison with female data', Journal of the Acoustical Society of America, 106 (2), pp. 1064-1077.

Hassan, Z.M. and B. Heselwood (Eds.) Instrumental Studies in Arabic Phonetics. Amsterdam: John Benjamins Publishing Company. 
Hassan, Z. M., Esling, J. H., Moisik, S. R. and Crevier-Buchman, L. (2011) 'Aryepiglottal trilled variants of $/ \mathrm{A}, \mathrm{h} / \mathrm{in}$ Iraqi Arabic', $17^{\text {th }}$ International Congress of Phonetic Sciences (ICPhS), Hong Kong (17-21 August), pp. 831-834.

Hawkins, S. and Stevens, K. N. (1985) 'Acoustic and perceptual correlates of the non-nasal nasal distinction for vowels', Journal of the Acoustical Society of America, 77 (4), pp. $1560-1575$.

Heselwood, B. (2007) 'The 'tight approximant' variant of the Arabic 'ayn ', Journal of the International Phonetic Association, 37, (1), pp. 1-32.

Heselwood, B. and Al-Tamimi, F. (2011) 'A study of the laryngeal and pharyngeal consonants in Jordanian Arabic using nasoendoscopy, videofluoroscopy and spectrography'. In Z.M. Hassan and B. Heselwood (Eds.). Instrumental Studies in Arabic Phonetics, pp. 101-128. Amsterdam: John Benjamins Publishing Company.

Hetzron, R. (1969) 'Two notes on Semitic laryngeals in East Gurage', Phonetics, 19, pp. 6981.

House, A. S. and Stevens, K. N. (1956) 'Analog studies of the nasalization of vowels', Journal of Speech and Hearing Disorder, 21, (2), pp. 218-232.

Hyman, L. M. (1972) Nasals and nasalization in Kwa. Studies in African Linguistics, 3(2), pp.167-205.

Ingham, B. (1982) North East Arabian Dialects. Boston and London: K. Paul International.

Iseli, M., Shue, Y.-L. and Alwan, A. (2007) 'Age, sex, and vowel dependencies of acoustic measures related to the voice source', Journal of the Acoustical Society of America, 121(4), pp. 2283-2295.

Kawasaki, H. (1986) 'Phonetic explanation for phonological universals: the case of distinctive vowel nasalization'. In Ohala, J. J. \& Jaeger, J. J. (Eds.), Experimental Phonology, Cambridge, MA: MIT Press, pp. 81-103. 
Keating, P. A., \& Esposito, C. (2007) 'Linguistic voice quality', University of California Los Angeles Working Papers in Phonetics, (105), pp. 85-91.

Kent, R. D. and Read, C. (1992) The Acoustic Analysis of Speech. San Diego: Singular Publishing Group, Inc.

Klatt, D. H. and Klatt, L. C. (1990) 'Analysis, synthesis, and perception of voice quality variations among female and male talkers', Journal of the Acoustic Society of America, 87, (2), pp. 820-857.

Kuang, J., \& Keating, P. (2012) 'Glottal articulations of phonation contrasts and their acoustic and perceptual consequences'. UCLA Working Papers in Phonetics, 111, pp. 123-161. Retrieved from http://escholarship.org/uc/item/0n76t3hn.

Kuznetsova, A. Brockhoff, P.B. and Christensen, R.H.B. (2016) lmerTest: Tests in Linear Mixed Effects Models. $\mathrm{R}$ package version 2.0-32. https://CRAN.Rproject.org/package $=$ lmerTest

Ladefoged, P. (2003) Phonetic Data Analysis: An Introduction to Fieldwork and Instrumental Techniques. Malden, MA, USA: Blackwell Pub.

Laradi, W. J. (1983) Pharyngealization in Libyan (Tripoli) Arabic: An Instrumental Study PhD Thesis. University of Edinburgh.

Laufer, A. and Condax, I. D. (1979) 'The epiglottis as an articulator', $9^{\text {th }}$ International Congress of Phonetic Sciences, Copenhagen, pp. 50-56.

Laufer, A. and Condax, I. D. (1981) 'The function of the epiglottis in speech', Language and Speech, 1 (Part 1), pp. 39-61.

Laver, J. (1980) The Phonetic Description of Voice Quality. Cambridge: Cambridge University Press.

Laver, J. (1994) Principles of Phonetics. Cambridge: Cambridge University Press. 
Le, S., Josse, J., Husson, F. (2008) FactoMineR: An R Package for Multivariate Analysis. Journal of Statistical Software, 25(1), 1-18.

Lenth, Russell V. (2016). Least-Squares Means: The R Package 1smeans. Journal of Statistical Software, 69(1), 1-33.

Ligges, U. and Mächler, M. (2003) Scatterplot3d - an R Package for Visualizing Multivariate Data. Journal of Statistical Software, 8(11), 1-20.

McCarthy, R.J. and Raffouli, F. (1964) Spoken Arabic of Baghdad: Anthology of Texts. Beirut: Librairie Orientale.

MacCurtain, F. (1981) Pharyngeal Factors Influencing Voice Quality. PhD Thesis. University of London.

Maeda, S. (1982) 'The Role of the Sinus Cavities in the Production of Nasal Vowels', Institute of Electrical and Electronics Engineers International Conference (IEEE) on Acoustics, Speech and Signal Processing (ICASSP). Paris, France, pp. 911-914.

Mahdi, Q. R. (1985) The Spoken Arabic of Basrah, Iraq: A Descriptive Study of Phonology, Morphology and Syntax. PhD Thesis. University of Exeter.

Matisoff, J.A. (1975) 'Rhinoglottophilia: the mysterious connection between nasality and glottality’. In C. Ferguson, L.H. Hyman, and J.J. Ohala (eds.). Nasalfest: Papers from a Symposioum on Nasals and Nasalisation. University of Stanford, pp. 265-287.

Obrecht, D.H. (1968) Effects of the Second Formant on the Perception of Velarization Consonants in Arabic. Berlin \& New York: Mouton De Gruyter.

Ohala, J. J. (1975) 'Phonetic explanations for nasal sound patterns', in Ferguson, C. A., Hyman, L. M. and Ohala, J. J. (eds.) Nasalfest: Papers from a Symposium on Nasals and Nasalization. University of Stanford, pp. 289-316.

Ohala, J. J. and Ohala, M. (1993) 'The phonetics of nasal phonology: theorems and data', Phonetics and Phonology, 5, (Nasals, Nasalization, and the Velum), pp. 225-249. 
Rabin, C. (1951) Ancient West-Arabian. London: Taylor's Foreign Press.

R Core Team (2016) R: A language and environment for statistical computing. R Foundation for Statistical Computing, Vienna, Austria. URL https://www.R-project.org/.

Sampson, R. (1999) Nasal Vowel Evolution in Romance. Oxford: Oxford University Press.

Stevens, K. N. (2000) Acoustic Phonetics. Cambridge, Mass.: MIT Press.

Trittin, P. J. and de Santos y Lléo, A. (1995) 'Voice quality analysis of male and female spanish speakers', Speech Communication, 16, pp. 359-368.

Wickham, H. ggplot2: Elegant Graphics for Data Analysis. Springer-Verlag New York, 2009.

Yeou, M. and Maeda, S. (2011) 'Airflow and acoustic modelling of pharyngeal and uvular consonants in Moroccan Arabic'. In Z. Hassan and B. Heselwood (Eds.). Instrumental Studies in Arabic Phonetics, pp. 141-164. Amsterdam: John Benjamins Publishing Company.

Younes, M. (1993) 'Emphasis spread in two Arabic dialects'. In Eid, M. and Holes, C. (eds.). Perspectives on Arabic linguistics V. Amsterdam: John Benjamins pp, 119-145.

Zeroual, C., Esling, J. H. and Crevier-Buchman, L. (2008) 'The Contribution of Supraglottic Laryngeal Adjustments to Voice: Phonetic Evidence from Arabic', Logopedics Phoniatrics Vocology, 33 (1), pp. 3-11. 